\title{
Ising models of quantum frustration
}

\author{
R. Moessner and S. L. Sondhi \\ Department of Physics, Princeton University, Princeton, NJ 08544, USA
}

(February 6, 2008)

\begin{abstract}
We report on a systematic study of two dimensional, periodic, frustrated Ising models with a quantum dynamics introduced via a transverse magnetic field. The systems studied are the triangular and kagome lattice antiferromagnets, fully frustrated models on the square and hexagonal (honeycomb) lattices, a planar analog of the pyrochlore antiferromagnet, a pentagonal lattice antiferromagnet as well as a two quasi one-dimensional lattices that have considerable pedagogical value. All of these exhibit a macroscopic degeneracy at $T=0$ in the absence of the transverse field, which enters as a singular perturbation. We analyze these systems with a combination of a variational method at weak fields, a perturbative Landau-Ginzburg-Wilson (LGW) approach from large fields as well as quantum Monte Carlo simulations utilizing a cluster algorithm. Our results include instances of quantum order arising from classical criticality (triangular lattice) or classical disorder (pentagonal and probably hexagonal) as well as notable instances of quantum disorder arising from classical disorder (kagome). We also discuss the effect of a finite temperature, as well as the interplay between longitudinal and transverse fields - in the kagome problem the latter gives rise to a non-trivial phase diagram with bond-ordered and bond-critical phases in addition to the disordered phase. We also note connections to quantum dimer models and thereby to the physics of Heisenberg antiferromagnets in short-ranged resonating valence bond phases that have been invoked in discussions of high-temperature superconductivity.
\end{abstract}

PACS numbers: 05.50.+q, 75.10.-b, 75.10.Jm, 75.30.Kz

\section{INTRODUCTION}

The study of frustrated magnetic systems began a half century ago with the realization by Wannierl and Houtappel 3 that the antiferromagnetic Ising model on a two-dimensional triangular lattice does not order down to the lowest temperatures and exhibits a finite entropy density even at $T=0$, in contrast to the naive expectation from the third law of thermodynamics. These two observations are related, and can be traced to the frustrated nature of the couplings even at the level of a single plaquette (Fig. 1) where one sees that it is not possible to minimize the energy of all three bonds simultaneously leading to six (instead of the two for ferromagnets) minimum energy configurations. From this we may infer a macroscopic entropy density for the triangular lattice at $T=0$ and rationalize the absence of any ordering by the easy accessibility of a large number of configuration at all temperatures. 3

The twin observations, of a non-vanishing entropy and lack of order, when contrasted with the ordering transition and small number (two) of ground states in the ferromagnetic Ising model on the same lattice, typify the striking behavior of such "maximally" frustrated classical models whose catalog, by now, includes also models with continous spins such as the Heisepherg magnet on the three dimensional pyrochlore lattice. 5 . Other, by definition, less frustrated models exhibit non-obvious phase transitions at finite temperatures. These may involve a singular privileging of the ground state manifold as a whole as in the case of the XY magnet on the kagome lattice $\mathrm{l}$ or, more typically, the elegant phenomenon of "order by disorder" 10 in which ground states that per- mit especially soft fluctuations about themselves are selected entropically at finite temperatures.

This paper is concerned with the fate of such frustrated models, especially the maximally frustrated ones, when they are endowed with a quantum dynamics at $T=0$. The canonical problems of this kind are $S=1 / 2$ antiferromagnetic quantum Heisenberg models on triangular 11 and kagome lattices 12 which have both attracted a considerable amount of interest. While one can think of these as quantized versions of classical Heisenberg models, it is instructive instead to follow Anderson and Fazekas 13 and think of them as perturbed variants of their Ising limits, which are manifestly frustrated. In these particular cases, the perturbation is the XY exchange and as it does not commute with the Ising pieces, it introduces a quantum (two-spin) dynamics into the frustrated problem. This perspective, in turn, suggests consideration of a more general class of models with alternative perturbations introducing a quantum dynamics instead.

In this paper we report on a systematic study of frustrated Ising models perturbed by transverse fields - as these introduce a single spin dynamics, they are evidently the simplest models in the extended class. 14 A short partial, account of this work has appeared previously 16 This paper covers a broader range of issues (and lattices) and supplies many of the details left out in the short account. It also provides a compendium of results we have obtained in the course of our work, which we hope will be of some use to people undertaking further study in this field. It is perhaps worth noting that this simplicity does make the models more tractable, which is generally true for transverse field Ising models and accounts for their ubiquity in quantum statistical mechanical con- 
texts. Much of this work is reviewed in Ref. 17, which also includes some previous work on one-dimensional frustrated chains. More recent studies include the use of the unfrustrated model as a paradigm of quantum critical behavior 18 and the treatment of random versions by asymptotically exact real space renormalization techniques near infinite disorder fixed points 19

A second motivation for studying these models is the possibility of direct experimental realization. Ising systems exist and in the case of $\mathrm{LiHoF}_{4}$ and its kin, transverse fields have been used to tune between phases in clean and disordered systems.20 A second family of Ising systems consists of stacked triangular lattices, strongly coupled along the stack, reviewed at length in Ref. 11 . While these have an intimate connection to the single triangular lattice in a transverse field via its euclidean representation, it would be interesting in future work to consider the effect of a transverse field on them. Meanwhile we would encourage our experimental colleagues to search for a triangular or kagome Ising antiferromagnet which are both, as we shall show in this paper, exemplars of very different physics that can arise in frustrated quantum systems.

Finally, there is the possibility of finding systems where there is a local Ising degree of freedom that is not itself an Ising spin. One such connection, which is currently the subject of intense interest, is an exact mapping from frustrated transverse field Ising models 6 to quantum dimer models of the short-range resonating valence bond state conjectured a while back by Anderson 21 and oft mentioned in discussions of the cuprate superconductors; in this context the Ising models appear more naturally as their dual, Ising gauge theories.22 28

Turning now to the physics of the models themselves, we note that the introduction of quantum fluctuations can be expected to lead to a variety of behaviors much as in the case of thermal fluctutations catalogued above. Indeed, in the quantum case, the singular character of the fluctuations is manifest in that even infinitesimal strength perturbations lead to a non-trivial problem of degenerate perturbation theory in a macroscopically degenerate manifold and can therefore be expected to lift the degeneracy and select a much smaller number of ground states. Consequently there must be a discontinuity in the entropy and ground state correlations at zero quantum coupling and $T=0$. We should emphasize that degenerate perturbation theory problems are not themselves perturbative, especially for macroscopic degeneracies. The most notable example of this is the quantum Hall problem where the degeneracy of a partially filled Landau level is lifted by the interaction and disorder in ways that lead to an incredibly complex phase diagram. Part of the interest of studying a diversity of perturbations of frustrated magnets is the prospect of generating at least a fraction of this complexity.

Two possibilities are generic at small quantum couplings: a) a quantum version of order by disorder 10 in which a broken symmetry state constructed out the the degenerate manifold is selected and b) a quantum version of the disordered or (cooperative) paramagnetic possibility in which the ground state correlations remain short ranged and might be expected to lead to the opening of a gap in the spectrum. This latter possibility, which we have christened "disorder by disorder", was first suggested by Anderson and Fazekas 13 as a mechanism for obtaining spin-liquid states of the RVB kind. Further singularities can emerge at large quantum couplings. For single spin dynamics, such as the transverse field problems we study, there is neccessarily a paramagnetic phase at large couplings and at least one phase transition en route in cases of order by disorder. The nature of such phase transitions, which will be of some interest to us in this paper, can indeed be very unconventional.

Specifically, we study Hamiltonians of the form:

$$
H=\sum_{\langle i j\rangle} J_{i j} S_{i}^{z} S_{j}^{z}+\Gamma \sum_{i} S_{i}^{x}+h \sum_{i} S_{i}^{z},
$$

on a variety of one and two dimensional lattices (see Fig. 2). Here the $J_{i j}$ are nearest-neighbor exchange couplings with $\left|J_{i j}\right|=J$ and $\prod_{\text {plaquette }}\left(-J_{i j} / J\right)=-1, \Gamma$ is the strength of the transverse field, the $S^{a}$ are the Pauli spin operators and $h$ is the strength of a (classical) longitudinal, Ising symmetry breaking field.

The structure of this paper is as follows. We first introduce concepts and methods we have found useful in studying the model under consideration (Sects. II and III). These we apply in the remainder of the paper to a number of frustrated Ising models on different lattices which, between them, realise a wide range of classical and quantum properties. We conclude by discussing possible realisations of such models.

In detail, in Sect. I1, we use a mapping of the $d$ dimensional quantum model to a $d+1$ dimensional classical model to derive a general criterion for the existence of a quantum ordering transition, and we use this mapping to obtain a quantum Monte Carlo algorithm. This algorithm is free of any sign problems and is one of the attractions of studying this class of models - augmented by a cluster method appropriate for our problems, we have used it at several points in this work.

In Sect. III, we consider a three-leg ladder that has considerable pedagogical value and allows us to introduce a weak coupling $(\Gamma / J \ll 1)$ variational analysis and a strong coupling Landau-Ginzburg-Wilson analysis that will be our principal analytic tools in the remainder of the paper. Sect. IV reports results on the somewhat baroque pentagonal lattice antiferromagnet which turns out to have an intimate connection with the ladder described previously. Its classically disordered state gives way to quantum order. In the next three sections (Sect. V, V1, VII we discuss three models: the triangular lattice antiferromagnet, the fully frustrated square lattice and the "two dimensional pyrochlore" lattice (see Fig. 2), respectively. These exhibit critical classical correlations in their ground state manifolds, and undergo 
ordering transitions, in accordance with the ordering criterion derived in Sect. II. The latter also exhibits two unconventional critical phases.

Next we turn to classically strongly disordered systems. Sect. VIII deals with the kagome lattice antiferromagnet which is a notable instance of disorder by disorder and also exhibits a highly non-trivial phase diagram when both transverse and longitudinal fields are present. Sect. IX] deals with a one dimensional Ising quantum disordered magnet, the sawtooth chain, the classical version of which is the ultimate cooperative paramagnet. Finally, Sect. X analyzes the fully frustrated honeycomb lattice which appears to exhibit a fairly complex pattern of ordering driven by quantum fluctuations as well as a non-trivial, $O(4)$ phase transition.

At various places in the paper we discuss connections to quantum height or dimer models. Via the latter, we find a connection to frustrated, valence bond, phases of Heisenberg magnets that has been of interest starting from the oppposite end 2325 and more recently starting from d-wave superconductors 27,26 As noted before, we are hopeful that this represents a more general possibility of realizing the models we study here in other contexts where frustration is present and it is possible to focus on a local Ising degree of freedom. We close with a brief recapitulation of our themes in the summary section.

\section{A CRITERION FOR ORDERING AND A METHOD FOR QUANTUM MONTE CARLO}

In this section we map our $d$-dimensional quantum magnet onto a $d+1$-dimensional ferromagnetically stacked, classical magnet. This mapping allows use to derive some qualitative features of the quantum ordering process, and also to make contact with the existing literature on stacked magnets. In addition, it enables us to devise a quantum Monte Carlo code free of the sign problem for general classical terms in the Hamiltonian.

\section{A. Mapping onto the stacked magnet}

We proceed using the Suzuki-Trotter formalism,29,30 to determine the precise correspondence between the quantum $d$ and classical $d+1$ dimensional models. Specifically, the partition function of the transverse-field Ising model is

$Z=\operatorname{Tr} e^{-\beta H}=\operatorname{Tr} \exp \left\{-\beta\left(v V\left(\left\{S_{i}^{z}\right\}\right)+\Gamma \sum_{i} S_{i}^{x}\right)\right\}$

where $\beta \equiv 1 / k_{B} T$. For generality, we have introduced the notation $V\left(\left\{S_{i}^{z}\right\}\right)$ for the 'classical' part of the Hamiltonian of strength $v$, i.e., $\left[V, S_{i}^{z}\right]=0$ for all $i$; in the simplest case, $V$ only consists of the exchange part of $H$, $v V=\sum_{\langle i j\rangle} J_{i j} S_{i}^{z} S_{j}^{z}$.
We now use a path integral representation of 2.1) where the insertion of a complete set of states effectively introduces an additional dimension of size $\beta$, we follow Suzuki's approach29.

$$
\begin{aligned}
Z= & \sum_{\left\{S_{i}\right\}}\left\langle\left\{S_{i}\right\}\left|\left(\exp \left(-a_{\tau} H\right)\right)^{N}\right|\left\{S_{i}\right\}\right\rangle \\
= & \sum_{n=1}^{\beta / a_{\tau}} \sum_{\left\{S_{i, n}\right\}}\left\langle\left\{S_{i, n}\right\}\left|\exp \left(-a_{\tau} H\right)\right|\left\{S_{i, n+1}\right\}\right\rangle \\
= & \sum_{n=1}^{\beta / a_{\tau}} \sum_{\left\{S_{i, n}\right\}} \exp \left(-a_{\tau} v V\left(\left\{S_{i, n}\right\}\right)\right) \times \\
& \times\left(\delta_{\left\{S_{i, n}\right\},\left\{S_{i, n+1}\right\}}^{(0)}+\frac{1}{2} a_{\tau} \Gamma \delta_{\left\{S_{i, n}\right\},\left\{S_{i, n+1}\right\}}^{(1)}+O\left(\left(a_{\tau} \Gamma\right)^{2}\right)\right)
\end{aligned}
$$

Here, we have introduced the imaginary time step $a_{\tau}$ and $n$ labels the coordinate of the extra dimension. The function $\delta^{(k)}$ is defined to be one if its arguments, the two (ordered) sets of spin configurations differ by $k$ entries, and zero otherwise.

We now establish an equivalence between the transverse field model and the classical Hamiltonian

$$
H_{d+1}=\sum_{\langle i j\rangle, n} K_{V}^{s} V\left(\left\{S_{i, n}\right\}\right)+\sum_{i, n} K^{\tau} S_{i, n} S_{i, n+1}
$$

by expressing the partition sum for the latter in terms of a transfer matrix, $T_{Z}$ :

$$
\begin{aligned}
T_{Z}= & \exp \left(K_{V}^{s} V\left(\left\{S_{i, n}\right\}\right)\right) \times \\
& \times\left(\delta_{\left\{S_{i, n}\right\},\left\{S_{i, n+1}\right\}}^{(0)}+\exp \left(-K^{\tau} / 2\right) \delta_{\left\{S_{i, n}\right\},\left\{S_{i, n+1}\right\}}^{(1)}+\right. \\
& \left.+O\left(\exp \left(-K^{\tau}\right)\right)\right) .
\end{aligned}
$$

The first term on the right hand side of the previous equation is to be understood as an exponentiated diagonal matrix.

¿From this, one can read off that the two partition functions will be equivalent if one chooses $a_{\tau} \Gamma / 2=$ $\exp \left(-K^{\tau} / 2\right)$ and $K_{V}^{s}=a_{\tau} v$. We note that continuous quantum evolution corresponds to the scaling limit $K_{V}^{s} \propto a_{\tau} \rightarrow 0, K^{\tau} \rightarrow \infty$, while maintaining

$$
2 e^{-K^{\tau} / 2} / a_{\tau}=\Gamma, \quad K_{V}^{s} \exp \left(K^{\tau} / 2\right) / 2=v / \Gamma .
$$

With the classical Hamiltonian $H_{d+1}$ (Eq. 2.4), the Ising spins interact in the spatial layers as they do in the analogous $d$ dimensional classical problem, but they are also coupled ferromagnetically in the additional (imaginary time) dimension. The dimensionless inverse of the quantum temperature, $\beta \Gamma$, is given by the extent $L^{\tau}$ of the system in the time direction: $\beta \Gamma=a_{\tau} \Gamma L^{\tau}=$ $2 \exp \left(-K^{\tau} / 2\right) L^{\tau}$. 


\section{B. A criterion for order by disorder}

We can look at the possibility of quantum ordering in a transverse field by studying the discretized partition function $Z_{\infty}\left(K^{\tau}\right) \equiv Z\left(K^{s} \rightarrow \infty, K^{\tau}\right)$ as an expansion in powers of $K^{\tau}$. In this limit, we force the spin configurations in each plane to be classical ground states. Taking the trace, $\operatorname{Tr}_{\mathrm{gs}}$ over these ground states gives

$$
Z_{\infty}\left(K^{\tau}\right)=\operatorname{Tr}_{\mathrm{gs}} \exp \left(K^{\tau} \sum_{i, n} S_{i, n} S_{i, n+1}\right)
$$

where the sum is over all ground-states for each layer. Expanding in small $K^{\tau}$, we obtain

$$
\begin{aligned}
Z_{\infty}\left(K^{\tau}\right)= & \operatorname{Tr}_{\mathrm{gs}}\left(1+\frac{1}{2}\left(K^{\tau}\right)^{2} \times\right. \\
& \left.\times \sum_{i, n} \sum_{j, n^{\prime}} S_{i, n} S_{i, n+1} S_{j, n^{\prime}} S_{j, n^{\prime}+1}+O\left(\left(K^{\tau}\right)^{4}\right)\right)
\end{aligned}
$$

where the linear term in $K^{\tau}$ is absent due to Ising symmetry. We can further express Eq. (2.8) as

$Z_{\infty}\left(K^{\tau}\right)=Z(L)^{L^{\tau}}\left\{1+\frac{1}{2}\left(K^{\tau}\right)^{2} \sum_{i, n}\left\langle S_{i, n} S_{i, n+1}\right\rangle^{2}+\ldots\right\}$

where the prefactor refers to the number of classical states in each layer of area $L^{2}$ and $L^{\tau} \equiv \beta / a_{\tau}$ is the number of layers. Then

$$
\begin{aligned}
Z_{\infty}\left(K^{\tau}\right)= & \exp \left(L^{2} L^{\tau} \mathcal{S}\right) \times \\
& \times\left\{1+\frac{1}{2}\left(K^{\tau}\right)^{2}\left(L^{2} L^{\tau}\right) \sum_{i}\left\langle S_{i, 0} S_{0,0}\right\rangle^{2}+\ldots\right\}
\end{aligned}
$$

where $\mathcal{S}$ is the classical ground-state entropy density. Therefore the effective free energy, $F_{\infty}=-\ln Z_{\infty} / \beta$ as a function of small $K^{\tau}$ is

$$
-\beta F_{\infty}=\left(L^{2} L^{\tau}\right)\left\{\mathcal{S}+\frac{\left(K^{\tau}\right)^{2}}{2} \sum_{i}\left\langle S_{i, 0} S_{0,0}\right\rangle^{2}+\ldots\right\}
$$

where the term $\left(L^{2} L^{\tau}\right)$ is a volume in space-time.

This yields the following powerful result. If the sum $I=\sum_{i}\left\langle S_{i, 0} S_{0,0}\right\rangle^{2}$ of the classical correlation function diverges, the free energy above is non-analytic as $K^{\tau} \rightarrow 0$, implying that the $K_{i j}^{s}=\infty$ is in a different phase than the disordered point $K^{\tau}=0$ for any $K^{\tau}>0$; this is indeed the case for the Ising triangular antiferromag net where $I_{t r i}$ contains a leading divergence of the form $\int d^{2} r(1 / \sqrt{r})^{2}$. In this case, one does indeed find quantum order by disorder (see Sect. $\mathrm{V}$ ).

However the situation is inconclusive for classically disordered antiferromagnets with exponentially decaying spin correlations at $T=0$; even though no single term in the series expansion, Eq. (2.11), becomes unbounded, the whole series may diverge because it is beyond its radius of convergence. There is therefore no clear link between classical and quantum disorder.

\section{Quantum Monte Carlo}

By simulating the stacked classical magnet, it is thus possible to gain information on the properties of the quantum system. We note that, thanks to the simplicity of our quantum dynamics, there is no sign problem to cope with for this class of models.

However, the scaling limit $K^{\tau} \rightarrow \infty$ does pose some technical problems. At large $K^{\tau}$, domain walls in the time direction become very rare, which leads to a divergent timescale in the Monte Carlo simulations. This problem can be remedied by employing a cluster algorithm, in which the attempted Monte Carlo moves consist not of flipping a single spin but rather rods of spins in the time direction. These rods can be chosen in a way which exactlycancels the inclement Boltzmann factor $\exp \left(-K^{\tau} / 2\right) 31$ It is with this method that we have carried out the simulations presented here, with an additional feature for the fully frustrated hexagonal lattice, (2 which is described there (see Sect. X).

It however turns out that the dominant source of error can be the remaining discretisation error. To see this, consider the case of a quantum ordered state at zero quantum temperature, $1 / \beta=0$, which corresponds to an infinite extension of the stacked model in the time direction. One can now imagine starting at an effectively infinite classical coupling, $K^{\tau}$. As $K^{\tau}$ is reduced, within the framework of the above mapping, one retains $1 / \beta=0$ but a discretisation error is introduced - which for sufficiently small $K^{\tau}$ will be serious enough to make the quantum order disappear as the classical magnet goes through its transition at a critical $K^{\tau}$. If one considers a finite $\beta$, one in practice has to trade off this error, which requires large $K^{\tau}$ against reaching a low quantum temperature, which nominally decreases with increasing $K^{\tau}$.

To quantify the discretisation error, we quote the parameter $\lambda \equiv \exp \left(k K^{\tau} / 2\right)$, (where $k$ is the multiplicity of the spin flip, being one for single-spin dynamics), a lengthscale characteristic of an isolated ferromagnetically coupled rod - the larger $\lambda$, keeping $\lambda / L^{\tau}$ fixed, the better. For fixed $L^{\tau}$, however, the optimal value of $\lambda$ depends on the correlation length in the time direction, which varies from system to system and which is larger the smaller the quantum gap.

In addition, the time for building up the clusters also becomes large at low temperature. This reflects the fact that the stacked classical magnet represents a rather inefficient way of doing the bookkeeping for the spin state. In the presence of only a few domain walls, it is probably su- 
perior to keep track of the domain walls themselves. This can be done in the framework of a continuous time algorithm, recommended for future use, which is described for example in Ref. 32 .

\section{METHODS USED AND THE FULLY FRUSTRATED THREE-LEG LADDER}

To introduce some of the concepts used repeatedly in this paper, let us first consider the toy model of the fully frustrated three-leg ladder depicted in Fig. 3. The interactions along the rungs are ferromagnetic, as are those along the outer two legs. The antiferromagnetic interactions along the middle leg make the ladder fully frustrated.

The classical ground states are those states which minimise the number of frustrated bonds. Since the bonds on the outer two legs belong to only one plaquette, it is not favourable to frustrate these. By contrast, frustrating one inner bond can put the two plaquettes it belongs to into the ground state. One finds that there are three sectors 33 of ground states, and their Ising reversed counterparts, depicted in Fig. 3. In all these states, the top and bottom legs are ordered ferromagnetically. The staggered sectors, with the spins on the top and bottom legs of opposite sign, contain only one ground state each; by contrast, the columnar sector has an extensive zerotemperature entropy per rung of $\mathcal{S} \equiv S_{0} /\left(N k_{B}\right)=G$, where $G=(\sqrt{5}+1) / 2$ is the golden mean, which can be obtained by a transfer matrix approach.

Since each plaquette has exactly one frustrated bond, which is shared with a neighbouring plaquette, one can represent the ground states by placing dimers onto the frustrated bonds. This gives rise to a hardcore dimer covering of the dual lattice, which in this case is a twoleg ladder. Note that such a mapping is possible for all fully frustrated models in which the elementary plaquettes are arranged to share bonds. It does not, however, exist for all lattices; for instance, if the plaquettes share sites rather than bonds, as is the case for the kagome lattice, the hardcore nature of the dimer model is lost. The advantage of this mapping is that, up to a global spin reversal, there is a one-to-one correspondence between the ground states and the hardcore dimer coverings. Therefore, restricting the full Hilbert space to that of the dimer coverings yields a natural implementation the projection onto the ground state. Below, we will present a derivation of the transverse field Hamiltonian restricted to the dimer (ground-state) manifold.

\section{A. The action of the transverse field - mapping onto a quantum dimer Hamiltonian}

In the $S^{z}$ basis, the transverse field operator $\Gamma S^{x}$ is a spin-flip operator, $(\Gamma / 2)\left(\begin{array}{ll}0 & 1 \\ 1 & 0\end{array}\right)$. The leading-order quantum dynamics within the ground state manifold is therefore that of single spin flips connecting different ground states - we need to consider multiple spin flips only in cases where single spin flips are nowhere possible, as will be the case in some of the examples discussed further down.

Flipping a spin without leaving the ground state manifold is possible only if the spin is part of the same number of satisfied bonds as frustrated ones, or, in other words, if it experiences zero net exchange field. We refer to such spins as flippable spins. In dimer language, such a spin is at the centre of a dimer plaquette of the form $\mathbb{l}$ or $\mathbf{二}$, and flipping the spin exchanges the frustrated and the satisfied bonds and thus corresponds to the elementary dimer move $\mathbf{l} \leftrightarrow$ 二.

The two staggered sectors of the ground state contain only the two staggered dimer states. The states in the columnar sector can be obtained starting from the columnar dimer state by repeated application of the elementary plaquette move $1 \backslash \rightarrow$ 二.

Therefore, in the staggered ground-state sectors the transverse field has no effect since no elementary dimer moves are possible - indeed, there are no degenerate states which it can mix at $T=0$ at any finite order of the perturbation theory. This is different in the main sector, where the transverse field lifts the macroscopic degeneracy and promotes a particular linear combination of the classical ground states to the true, quantum ground state.

In fact, it is now apparent that the transverse field Hamiltonian, restricted to the classical ground state manifold, in dimer language can be written as

$$
H_{Q D M}=-t(|\bar{\Xi}\rangle\langle\mathbf{l} \mathbf{l}|+\text { h.c. })+v(|\vec{\Xi}\rangle\langle\vec{\Xi}|+| \mathfrak{l} \mathbf{l}\rangle\langle\mathbf{l}|),
$$

where the kinetic term with $t=\Gamma / 2$ is generated by the transverse field. We have added a diagonal term with coefficient $v$, which is zero for the transverse field problem but which will be useful later on.

Note that this Hamiltonian has only nonpositive offdiagonal matrix elements, so that the Perron-Frobenius theorem can be used to predict a nodeless quantum ground state, which means that in it, the amplitudes of all the configurations can be chosen to be real and nonnegative.

Since a ground state and its Ising reversed counterpart both get mapped onto the same dimer states, we have to show that the dimer ground state is in fact the same as the transverse field ground state. 34 First we split the ground state manifold into two submanifolds, namely those containing symmetric and antisymmetric combinations of Ising-reversed pairs of states. The transverse field 
Ising Hamiltonian does not connect these submanifolds, so that it is block-diagonal.

All entries in the symmetric block continue to be of the same (negative) sign so that the ground state in this block continues to be nodeless. Since the full Hamiltonian is block-diagonal, the state obtained by combining this ground state with a null state in the antisymmetric block continues to be a nodeless eigenstate of the full problem. Since there is only one nodeless eigenstate, the state obtained by translating the dimer ground state into the spin ground state is the ground state of the transverse field problem.

The entries in the antisymmetric sector have the same modulus as in the symmetric one; however, they need not all have the same sign. If we pick one spin state for each dimer state and collect those in the up manifold, and their spin-reversed counterparts into the down manifold, an entry will be negative when the transverse field connects members of the up and down manifolds. The states can be sorted in a way, e.g. by magnetisation, that the fraction of negative entries vanishes in the thermodynamic limit. Whether the ground states in the two sectors in the thermodynamic limit are degenerate then depends on whether the wavefunction has substantial support on the states at zero magnetisation, in which case they are not, or whether it is localised away from them, in which case they are. The example of the threeleg ladder is special in that the up and down sectors can be chosen to be entirely disconnected, so that an exact degeneracy trivially arises.

The connection between transverse field Ising model and quantum dimer model we have established is useful for several reasons. It affords some insight into the structure of the problem we are studying in that it provides a natural deformation of the transverse field Ising model by switching on the potential term, i.e. by choosing a nonzero $v$. The cases $|v| \gg t$ are easily solved and can therefore provide two anchors of the phase diagram containing the point we are interested in.

Moreover, there is the special point $v=t$, known as Rokshar-Kiyelson (RK) point, after the inventors of the model 22 where the (nodeless) quantum ground state is an equal-amplitude superposition of all classical ground states. Therefore, operators which are diagonal in the dimer basis provide precisely the expectation values of the corresponding classical operators. In this spirit, switching on an infinitesimal transverse field implies jumping the finite distance from $v=t$ to $v=0$ in the Rokshar-Kivelson model (Fig. 5), a manifestation of the fact that the transverse field perturbation is nonanalytic. Deciding the ordering pattern of the transverse field problem can therefore be accomplished if one can show that the RK and the transverse field points are in the same phase.

Moreover, the transverse field problem in itself provides a new perspective on the Rokshar-Kivelson quantum dimer model, which was proposed as a model of Anderson's (short-range) resonating valence bond (RVB) physics.21 It can be derived for a Heisenberg antiferromagnet: the perturbative derivation uses the nonzero overlap between different dimer configurations as expansion parameter. This model is useful in a regime where the Heisenberg model is in a phase dominated by valence bonds. The study of a transverse field Ising model on a frustrated lattice can thus be used to gain insight into the behaviour of Heisenberg magnets on the dual lattice. This fact has been used by the present authors to identify a bona-fide short-range RVB phase on the triangular lattice,28 a goal that had proven to be elusive on the square lattice, for which the model was originally formulated.

Finally, we note parenthetically that for the mixed bond models, the application of a longitudinal field is somewhat arbitrary as there is a (gauge/Mattis) freedom of which bonds to call antiferromagnetic and which ferromagnetic, as long as the odd condition is met. Thus any state in the gauge invariant dimer representation can be (up to topological restrictions) be represented by a maximally polarised (long-range ordered) spin configuration, irrespective of the nature of the dimer correlations. In the models where all bonds can be chosen to be antiferromagnetic, this choice defines the natural gauge and thus makes the application of a longitudinal magnetic field unambiguous.

\section{B. Maximally flippable states}

We now present a heuristic argument which is variational in spirit to generate a candidate state for selection by the transverse field. This state is the one which can gain the most energy from the transverse field on account of being composed around a backbone configuration having the strongest fluctuations possible.

To start, we note that in order to gain energy from the transverse field, spins have to have a component pointing in the $x$-direction, which in the $S^{z}$-basis goes along with the component of the form $[|\uparrow\rangle+|\downarrow\rangle] / \sqrt{2}$. Those states in which most of the spins can be flipped and hence put in a superposition of up and down states thus stand to gain most energy from the fluctuations induced by the transverse field. We therefore identify the configuration with the largest number of flippable spins ("maximally flippable state") as the backbone of our candidate ground state. The actual ground state naturally includes fluctuations around the maximally flippable configuration, since it is these which lead to the energy gain in the first place.35 The energy gain due to alignment of the spins along the transverse field in the quantum model is an entropic contribution to the free energy in the classical, stacked model.

As an aside, we note that the classical correlations provide the simplest first guess at the maximally flippable configurations and hence at the ordering pattern. This is because configurations with many flippable spins have a 
large number of neighbouring configurations which differ only by a few spin flips. The correlations they incorporate, even when not leading to long-range order, can thus already be visible in the classical average where all ground states are accorded equal weight.

For our three-leg ladder, the maximally flippable configuration is the columnar one depicted in Fig. 3, since there, all spins on the middle row (all of which point up) are flippable. We therefore expect to find a state which has a ferromagnetic moment even in the $z$-direction in addition to the polarisation in the $x$-direction.

The selected state can incorporate structure in addition to that apparent from the maximally flippable configuration. This follows from the fact that although the backbone configuration maximises the number of flippable spins, in fact not all the spins are independently flippable. In the three-leg ladder, for example, flipping a spin on the middle leg precludes flipping its neighbours, so that in effect only half the spins are independently flippable. One can therefore give two different recipes for constructing the quantum state.

To contruct the first type of state, we take all the flippable spins and polarise them in the $x$-direction disregarding the ground-state constraint. Next, we reinstate the ground-state condition by projecting out those components of the state which are not contained in the classical ground-state manifold. We call this state the uniform state since it treats all the flippable spins on the same footing.

The second type of state is obtained by identifying the largest set of independently flippable spins and polarising those in the $x$-direction. Since, starting from a maximally flippable configuration, there are typically several choices for which set of flippable spins to polarise, we call this state the hierarchical state. It will have a lower symmetry than the maximally flippable configuration unless all flippable spins are independently flippable.

The distinction between the uniform and the hierarchical states will turn up in several times in this article, and it arises quite naturally in other approaches. Note that this approach suggests yet another class of alternative candidate configurations, namely those which do not have the maximum number of flippable spins but nonetheless maximise the number of spins which are flippable independently.

The variational states thus obtained have the shortcoming that all their component configurations are allocated equal weight. This is clearly not optimal, since the ground state will in any case place maximal weight on the individual, maximally flippable configurations. Within a more elaborate variational framework, weights could be accorded to the configurations depending on their number of flippable spins.

In summary, the flippability approach suggested here identifies a 'saddle point' - the maximally flippable configuration - that will be favoured due to the fluctuations around it, which are 'softer' than those around other configurations. We expect it to work as long as the actual quantum wavefunction is concentrated on the maximally flippable and nearby configurations. It will break down if the wavefunction has the bulk of its support elsewhere, i.e. on configurations unrelated to the maximally flippable one.

This point can be made more intuitively by considering the degenerate perturbation theory as a hopping problem. Each classical ground state configuration defines a point in the ground-state manifold. The transverse field, by flipping spins, connects different configurations, thus endowing the ground-state manifold with a graph structure. The perturbation theory can be thought of as a hopping problem on the graph thus defined. The large weight of maximally flippable configurations follows from their high coordination, and the magnetic ordering transition corresponds to a localisation transition in the hopping problem 36

These ways of thinking are in close correspondence to the case of thermal order by disorder 6 There, thermal fluctuations (out of the ground state manifold) provide a large entropic weighting to the states allowing the softest fluctuations. These states are then selected as $T \rightarrow 0^{+}$ provided their enhanced weight is not swamped by the combined fluctuational and configurational entropy of the less favoured states 37 However, when the thermal fluctuations increase in strength with increasing temperature, they destroy the order which they were instrumental in establishing in the first place, as happens for $\Gamma$ large in our problem, as discussed in the following paragraphs.

\section{The opposite limit: Landau-Ginzburg analysis for $\Gamma \gg \mathbf{J}$}

In addition to doing the (hard) degenerate perturbation theory for small transverse fields, we can use an alternative approach for determining the state of the quantum magnet which is made tractable by virtue of the simplicity of the transverse-field term. Consider the problem where the relative sizes of exchange and transverse field are inverted, namely where $\Gamma \gg J$. In the limit $J / \Gamma=0$, the ground state is a simple paramagnet in the $S^{z}$ basis: all spins are polarised along the $+x$-direction explicitly selected by the field. In addition, it is gapped: the lowest excitations are spin flips which each cost an energy of $\Gamma$; this makes it possible to perturb about this state by switching on a weak exchange. This contrasts to the case where the transverse field is replaced by an XY exchange. Here, the large $J_{X Y}$ problem is not exactly soluble, and so it is not possible to perturb about it - indeed, it may even be gapless.

As the perturbing $J$ is switched on in addition to the transverse field, the excitations acquire a dispersion, typically - but not always - already to first order in $J / \Gamma$. The dispersion to first order is simply given by the Fourier transform of the interaction matrix of the lattice. For sufficiently large $J$, there can be an ordering transition, 
which corresponds to a macroscopic occupation of the softest mode(s).

To generate the state to which the leading transition takes place, one has to combine knowledge of the soft modes with lattice symmetry considerations to construct a Landau-Ginzburg-Wilson action. This program is carried out in detail for the fully-frustrated hexagonal magnet below and follows the york on layered magnets by Blankschtein and coworkers. 38 39

The combinations of the soft modes dictated by symmetry considerations yield the ordering pattern which is established as $J / \Gamma$ is increased. In addition, by also analysing the Landau-Ginzburg-Wilson action with standard methods of the renormalisation group, one can obtain information on the nature of the transition into the ordered configuration, and it turns out that these transitions are generally not Ising transitions, as the most naive guess would suggest. By analysing the potential presence of dangerously irrelevant terms in the action, one can even guess at further symmetry breaking.

This analysis of course has the usual limits associated with mean-field theories. Most important in this context is the possibility of further phase transitions out of the ordered phase before we reach the regime of infinitesimal transverse fields, where the 'small' parameter $J / \Gamma \rightarrow \infty$. Another scenario is the absence of any phase transition, so that the magnet remains disordered at all couplings. Although the excitation dispersion may soften at particular points in the Brioullin zone, fluctuations may be sufficiently strong to prevent ordering at any coupling. However, even in this situation, the large $\Gamma$ approach may be used successfully if the expansion in powers of $J / \Gamma$ is carried to sufficiently high order and combined with a non-perturbative analysis such as that given by the use of Pade approximants. In Ref. 40, this program has been carried out for the sawtooth chain (see Fig. 2).

It is worth pointing out that this approach presents, ultimately, a soft-spin analysis in that the size of the ordered moment can vary from site to site so that, as the mode amplitude increases, non-linearities become important. However, even for our hard Ising spins, a difference in the size of the ordered moment does have a meaning. As an illustration, consider the uniform state of the threeleg ladder, defined above. Here, every spin on the middle row is fluctuating and thus has $\left|\left\langle S_{z}\right\rangle\right|<1 / 2$, whereas the remaining spins have $\left\langle S_{z}\right\rangle=1 / 2$, being fully polarised along the $z$-axis. This difference is indeed found in the large $\Gamma$ analysis and can thus be interpreted as being due to the fluctuations induced by the transverse field.

In detail, for the three-leg ladder, the Fourier transform of the interaction matrix is (labeling the sites on the rung 1 to 3 from top to bottom)

$$
\frac{J}{2}\left(\begin{array}{ccc}
2 \cos (k) & 1 & 0 \\
1 & -2 \cos (k) & 1 \\
0 & 1 & 2 \cos (k)
\end{array}\right),
$$

from which we obtain a degeneracy in the dispersion relation. One minimal mode, with wavevector $q=0$, has eigenvector $(1,-2+\sqrt{6}, 1)$; the other has $q=\pi$ and eigenvector $(1,-2-\sqrt{6}, 1)$. Here, $q$ is the wavevector along the ladder, and the entries in the eigenvector denote the amplitudes of the top, middle and bottom sites $(\sqrt{6} \simeq 2.45)$.

This degeneracy is accidental in that the states are not related by any symmetry operations, and we have not encountered this effect in any of the more regular lattices we are studying. Quite generally, however, in lattices with sites of different coordination, the soft-mode analysis might suggest states which fare very poorly under the hard-spin constraint, as is the case for the $q=\pi$ mode in this example (see below). A more appropriate ordering pattern may nonetheless show up as an alternative, possibly only local minimum in the dispersion relation.

The $q=0$ mode can easily be identified as the actual ordering pattern (see below, exact diagonalisation), with a moment on the middle row reduced by the fluctuations. One reason the competing state loses out eventually is because it has a reduced moment on the sites which are in fact not allowed to fluctuate in the small- $\Gamma$ limit: there, the leading term in the perturbation theory flips spins with an equal number of frustrated and satisfied bonds, and the reduced moment sites in this state have odd coordination and are thus never flippable.

\section{Exact diagonalisation}

For the three-leg ladder, one can attack the transverse field problem by exact diagonalisation of the degenerate perturbation theory. Systems with up to fourteen rungs are easily accessible numerically. The results are depicted in Fig. 4. We do indeed find a state with ferromagnetic order along the centre row, as predicted by the flippability analysis. The selected state is the uniform columnar one, as the gap extrapolates to a finite value for large system sizes and hence the breaking of translational symmetry accompanying the hierarchical state is absent.

The infinitesimal transverse field generates a nonzero polarisation in the $x$-direction, corresponding to an ground-state energy per spin of $E_{0}=-(0.302 \pm 0.001)|\Gamma|$ in excess of the classical value. The finite moment $\left\langle S_{z}\right\rangle$ already present in the classical ground state average (where it equals $1 /(2(2 G-1)) \simeq 0.224)$, is discontinuously enhanced as $\Gamma$ is switched on. As the system size is increased, the maximally flippable state and its neighbours rapidly gain in weight, whereas at the other extreme, those states without flippable spins have zero amplitude in the ground state wavefunction.

These results tie in with the picture provided by the mapping onto a quantum dimer model. The selected columnar dimer phase, which extends all the way to $v=-\infty$, terminates at the RK point, beyond which it gives way to the staggered dimer phase, as depicted in Fig. 5.41 


\section{E. Relation to XY perturbations}

Whereas the transverse field induces single spin flips, an XY exchange flips neigbouring pairs of antialigned spins. For lattices with even coordination and all bonds antiferromagnetic, the XY flips are a subset of the two spin flips generated by two successive operations of the transverse field.

For lattices with odd coordination, however, the transverse field can never flip a spin without leaving the ground state manifold, so that the XY moves can show up as the leading terms, in order $\Gamma^{2} / J$, in the degenerate perturbation theory. These matrix elements are negative, corresponding to a ferromagnetic XY exchange. This will be of importance for the fully frustrated hexagonal lattice. Moreover, in the case of the pentagonal lattice, where inequivalent sites with even and odd coordination exist, the moves generated by an XY perturbation are entirely distinct from those generated by the transverse field.

\section{THE PENTAGONAL LATTICE}

The pentagonal lattice, depicted in Fig. 6, tiles the plane with (irregular) pentagons. It can be obtained from the hexagonal lattice by cutting each hexagon in half with a set of parallel lines ('cuts'). Classically, the Ising antiferromagnet on this lattice is disordered and has afinite ground-state entropy per spin of $\mathcal{S} / k_{B}=0.23442$ The ground states can be represented by a hardcore dimer model as described above.

The flippability analysis for this magnet is straightforward. To lowest order in $\Gamma$, only the spins along the cuts can be flipped since only they have even coordination. Therefore, the spins along the sawtooths follow an antiferromagnetic pattern, since the maximally flippable configuration (depicted in Fig. (6) has all frustrated bonds associated with spins on the cuts. This configuration is closely related to the fully frustrated three-leg ladder in that the spins along the cuts are effectively decoupled from the other spins in the system and the transverse field generates the same Hamiltonian as for the ladder. Thence, the ordering pattern along the cuts will be the $q=0$ pattern described above, with a $q=\pi$ modulation transverse to the cuts.

For completeness, we now perform the soft-mode analysis for this problem. The interaction matrix is

$$
\frac{J}{2}\left(\begin{array}{ccc}
2 \cos q_{x} & 1 & e^{-i q_{y}} \\
1 & 0 & 1+e^{-i q_{x}} \\
e^{i q_{y}} & 1+e^{i q_{x}} & 0
\end{array}\right)
$$

There is a line where the eigenvalues attain the global minimum $-J(1+\sqrt{3}) / 2$ at wavectors $(\pi, y)$. The eigenvectors $\left((-1-\sqrt{3}) e^{-i q_{y}}, e^{-i q_{y}}, 1\right)$ correspond to states with frustrated bonds on the sawtooths. As mentioned above, these do not lead to flippable spin arrangements to lowest order in the degenerate perturbation theory in $\Gamma$, and this state is therefore not competitive in the small $\Gamma$ limit. There is, in addition, one further local minimum $-J \sqrt{6} / 2$ at wavevector $(0, \pi)$. The eigenvector $(-2+\sqrt{6},-1,1)$ corresponds to the maximally flippable state (and agrees with the one given by the threeleg ladder). We therefore expect a phase diagram with at least three transitions coming from large $\Gamma$, namely first from the disordered into the 'sawtooth state' followed by a transition into the maximally flippable state, which finally terminates in the disordered classical phase at $\Gamma=0$. There may be a separate transition which selects one or a combination of the sawtooth states - we have not investigated this. The complicated structure of this phase diagram is of course to a large degree a consequence of the presence of inequivalent sites.

\section{THE TRIANGULAR ANTIFERROMAGNET}

The two-dimensional Ising antiferromagnet on the triangular lattice has power-law spin correlations and an extensive entropy $\left(\mathcal{S} / k_{B}=0.323\right)$ at $T=0$. 1. 2.4 We first describe a number of useful mappings and then discuss their implications.

\section{A. Mappings to height and dimer models}

In Fig. 月, we have displayed a particular spin configuration. The corresonding dimer configuration (also shown) is constructed as in Sect. III). The effect of the transverse field can easily be stated in terms of the dimer mapping in a more general setting than the one discussed in Sect. III). If we choose a closed loop of bonds on the dual lattice which are alternately empty and occupied by a dimer, and exchange the occupied with the empty bonds, this corresponds to flipping all the spins inside the closed loop. The leading order effect of the transverse field is therefore to produce such a rearrangement for the loop enclosing the smallest number of sites, and the relevant order in perturbation theory is given by the number of enclosed spins. The loop in question is typically the shortest closed loop of even length on the dual lattice. In this case, this is a loop of length six enclosing one site (see Fig. 7), but we will encounter the case of more than one enclosed site later on.

Next, we turn our attention to the mapping to a height model. The height configuration is given by the numbers in Fig. 7. The height variables are defined on the sites of the lattice. 43 Height differences are determined as follows. If one crosses a (no) dimer when going along a bond in a clockwise direction around a triangle pointing up, the height changes by $2(-1)$. If one goes the opposite direction, the height change is $-2(+1)$. One can easily check that this prescription has the property that the height change around the three sides of any triangle is 
zero. Moreover, any closed path can be decomposed into a sum of paths around individual triangles, and thus the height change between any two sites is indepent of the path chosen to go between them.

The existence of the height model has important ramifications for the classical model. Under a set of reasonable assumptions 43 the result follows that the ground state correlations are algebraic. Combined with our criterion for quantum ordering (Sect. IIB), one expects all magnets allowing a height mapping to order. The ordering correlations are then determined by the configuration which is as flat (in height space) as possible.

This raises the question under which conditions a height mapping exists. A set of sufficient conditions is that, firstly, the lattice be composed of bond sharing frustrated units (allowing a dimer model) and secondly, its dual lattice be bipartite. This is required for giving a set of consistent rules for computing height differences. In the cases we encounter below where these conditions are not met, we will see that a height mapping is absent, consistent with their disordered classical correlations.

\section{B. Ordering behaviour of the triangular IAFM}

We first study this problem for small transverse field with $h=0$. The maximally flippable configuration is the one depicted in Fig. 7. It has a unit cell of three sites (two spins pointing up and one down). This is also the flat (height) configuration, as well as being the state encounteredat $v=-\infty$ in the appropriate quantum dimer model:44 45

$$
H_{Q D M}=-t(|\vec{\nabla}\rangle\langle\boldsymbol{\Omega}|+\text { h.c. })+v(|\vec{\nabla}\rangle\langle\vec{\nabla}|+| \boldsymbol{\Omega}\rangle\langle\boldsymbol{\Omega}|) .
$$

In fact, this state has a net magnetisation since its three-sublattice strucutre is $(1,1,-1)$, with all spins pointing up being flippable. The uniform state will thus have a sublattice structure of the form $(a, a,-b)$, with $b>a$, whereas the hierarchical one will have $(c, 0,-c)$.

All the evidence therefore points towards a three sublattice quantum ordering pattern, with details to be determined. Such correlations were previously found by a Landau-Ginzburg-Wilson (LGW) analysis of ferromagnetically-stacked triangular lattices 38 applicable to our problem by virtue of the mapping presented in Sect. II A. This LGW analysis finds an action which is $\mathrm{XY}$ symmetric up to sixth order, where an XY symmetry breaking, six-fold clock term appears.

The resulting phase diagram in the temperature-field $(T-\Gamma)$ plane, depicted in Fig. 8, is quite remarkable. At $T=0$, the triangular TFIM undergoes a quantum phase transition which is in the 3 -d XY universality class, 38 where the clock term is dangerously irrelevant, so that the transition is into a phase with clock-symmetry breaking as well.

At finite temperature, however, the transverse field Ising model maps onto a classical stacked magnet of $f i$ nite size, $L^{\tau}$, in the imaginary time direction, so that its behaviour crosses over to being two-dimensional as the correlation length becomes comparable to $L^{\tau}$; in this regime, we thus have to consider the properties of a twodimensional XY model with a clock term. Here one finds 4 two finite-temperature Kosterlitz-Thouless transitions bordering an extended critical phase. The phase diagram in Fig. 8 is reliable in the region near the three dimensional XY transition, where the order parameters are small.

Depending on the sign of the six-fold clock term, the quantum ordered state is predicted to have the sublattice structure of either the uniform or the hierarchical state. In order to confirm the predicted ordering pattern and to find out the precise sublattice structure, we have carried out quantum Monte Carlo simulations on the triangular TFIM. The results are depicted in Figs. 9 and 10. A Bragg peak is clearly visible at $q_{x}=2 \pi / 3$, as expected. The sublattice structure of the form $(1,0,-1)$ is also clearly borne out, showing that the hierarchical state without a net moment is selected. Since the quantum dimer state at $v=-\infty$ is the uniform, magnetised state, this implies a transition for $v<0$. Preliminary studies by us indicate that such a transition is indeed present close to $v=0$. Finally, we note that a finite longitudinal field $(|h|<6 J)$ on its own selects the same uniform state.

\section{VILLAIN'S ODD MODEL: THE FULLY FRUSTRATED SQUARE LATTICE}

A great deal is known about the square FFIM, especially when we combine the knowledge about the different models it is equivalent to by virtue of the mappings presented above. It turns out to shadow the triangular IAFM in practically all important qualitative respects. Its classical ground-state entropy $\mathcal{S}=C / \pi \simeq 0.241,47$ where $C$ is Catalan's constant. Since it consists of bondsharing squares with a bipartite dual lattice, it is critical at $T=0$. The corresponding dimer model Hamiltonian is given by Eq. 3.1 and has a phase diagram like that pictured in Fig. 5. The columnar phase corresponds to the maximally flippable state which, in dimer language, is obtained by covering the square lattice with infinite dimer ladders with the configuration depicted on the right panel of Fig. 3. An LGW analysis 39 again finds an XY action with an XY symmetry breaking clock anisotropy at higher order, which, depending on its sign, selects either the uniform or the hierarchical (also known as plaquette) state.

This makes it clear that there is quantum ordering in the FFIM on the square lattice into a state with translational symmetry breaking. We have not studied this problem numerically ourselves but we refer the reader to literature on the ferromagnetically stacked magnet 39.48 and the quantum dimer model. 49.50 


\section{PYROCHLORE IN TWO DIMENSIONS: THE SQUARE LATTICE WITH CROSSINGS}

This lattice, also known as the checkerboard or twodimensional pyrochlore lattice, is made up of a square lattice with nearest neighbour interactions which has, in addition, crossing next-nearest neighbour interactions on alternate plaquettes as depicted in Fig. 11. The plaquettes with crossings are equivalent to tetrahedra in that they contain four sites all interacting equally with one another. Since these tetrahedra are arranged to share sites, as is the case in the pyrochlore lattice in three dimensions, from which it can in fact be obtained by a projection in a $\langle 111\rangle$-direction; for a picture, see Ref. 37b.

The ground-state condition for the classical Ising model is that each tetrahedron have zero magnetisation. There are six such states for each tetrahedron, each with two spins up and two down. The Ising model on the pyrochlore lattice is equivalent to the ice model 51 the ground state entropy of which was calculated exactly in two dimensions by Lieb:E2 $\mathcal{S} / k_{B}=\frac{3}{4} \ln \frac{4}{3} \approx 0.216$. This model can be mapped onto a six-vertex model (each vertex encoding one of the single-tetrahedron ground states), and hence onto a non-intersecting loop model, which in two dimensions guarantees the existence of a height model. As discussed above, this quite generally implies classically critical oround-state correlations, which have indeed been found. 33

Here, we present a short derivation of the height model which we then use to determine the quantum ordering behaviour; refer to Fig. 11. We define a set of onedimensional heights which reside on the vertices of the square lattice dual to the plaquettes without crossing interactions. Next, we assign an orientation (clockwise or anticlockwise) to each tetrahedron, so that neighbouring tetrahedra have opposite orientations. Since the lattice dual to the tetrahedra is bipartite, this can be done consistently. The rule for the height differences is as follows. If going from one site of the height lattice to another one passes over an up (down) spin, one increases (decreases) the height by one provided the spin was crossed in the direction given by the orientation of the tetrahedra it belongs to. In the opposite direction, one decreases (increases) the height by one. This generates a consistent assignment of the heights since going around a unit cell of the height lattice generates zero height difference by virtue of the ground-state two-up two-down condition, and because each closed path on the height lattice can be decemposed into a combination of such elementary loops.54

We next consider the action of a longitudianl field of strength $h$ in the absence of a transverse field. For $h<$ $2 J$, all ground states remain degenerate since they have zero net moment. At $h=2 J$, the applied field is strong enough to surmount the exchange field and it generates a spin-flop transition to a manifold of states with three spins up and one down in each tetrahedron. These states continue to have an extensive entropy but one which is reduced compared to the low-field value. In addition, the classical correlations in this regime, to which we allocate the name IM ('intermediate'-field regime), continue to be critical. This result follows from another mapping of those states onto a dimer model which in turn generates a height model.

This mapping is obtained as follows. Consider a dimer model on the square lattice dual to the tetrahedra. For each spin pointing down, place a dimer, centred on this spin, with its ends located at the points of the dual lattice denoting the centres of the tetrahedra the down spin belongs to. Since each tetrahedron has exactly one down spin, and since each spin is shared by two tetrahedra, each classical IM ground state generates a hardcore dimer covering of the (dual) square lattice and vice versa. Such a square-lattice dimer model can be mapped onto a height model, from which the criticality of the correlations in this regime follow. In the IM-phase, the entropy per spin is a quarter of the value found in the Villain model, as now there are four spins per dimer rather than one: $\mathcal{S} / k_{B}=0.073$.

We emphasize that the field leaves the entropy unchanged for a finite range of $h$ and then reduces to a lower value without eliminating it completely, while inducing a transition between two critical states; this again persists over a finite range of fields. At $h=6 \mathrm{~J}$, there finally occurs a transition to the fully polarised state.

Next, consider tilting the field such that $\Gamma \ll h$; this enables us to generate a perturbation theory controlled by the small parameter $\Gamma / h$. The transverse field induces matrix elements between the states corresponding to the classical ground state configurations. Both in the lowfield and the IM regime, the degeneracy is not lifted until fourth order in perturbation theory. The reason is that connecting two ground states requires flipping a closed loop of alternating spins which passes through an even number of sites of any tetrahedron. The shortest such loop has length four and is thus generated at fourth order in perturbation theory. The lower-order terms induce only a diagonal shift in the energies but this shift is the same for all states.

The short flippable loop, depicted in Fig. 11, has different interpretations in the two phases. For the low-field phase, it corresponds to changing the height of a plaquette whose four neighbouring heights are equal. In a manner analogous to the ground-state selection on the triangular lattice, this leads to the selection of the flat state in height language. In spin language, the flat state is a Neel state on the square lattice underlying the squarelattice with crossings.

In the IM phase, the quadruple spin-flip again leads to the RK model at $v=0$ and $t \propto \Gamma^{4} / J^{3}$ (see Eq. 3.1). This move connects different ground states since the total magnetisation of each tetrahedron remains unchanged and the effect of flipping the four spins is to generate the familiar dimer plaquette move $\| \leftrightarrows$ 二.

Carrying over the results from the fully frustrated 
square lattice, we expect the system to order into a flat (height) phase which corresponds to a columnar dimer phase. In spin language, this phase differs from the Neel low-field phase, from which it can be obtained by flipping half of the down spins, e.g. those which are located on the top-left hand corner of one sublattice of tetrahedra.

The complete phase diagram for this magnet in the $h-\Gamma$ plane can be constructed from this. The simplest phase diagram incorpoating our results is displayed in Fig. 12 .

\section{THE KAGOME LATTICE}

In the kagome Ising antiferromagnet, depicted in Fig. 2, the nearest-neighbor exchange couplings are uniform andantiferromagnetic. The ground-state entropy is finite, $55 \mathcal{S}_{\text {kag }}=0.502$, and is more than half of the maximum paramagnetic value $\left(\mathcal{S}_{\text {para }}=\ln 2\right)$ in contrast to the triangular case $\left(\mathcal{S}_{t r i}=0.323\right)$. Furthermore $\mathcal{S}_{k a g}$ is close in valye to that obtained by the Pauling approximation,5356 $\mathcal{S}_{\text {Pauling }}=\ln 2+\frac{2}{3} \ln \frac{3}{4} \approx$ 0.501 , where the triangles are considered independently; this suggests that spin-spin correlations in the kagome IAFM are extremely weak. This is indeed the case, and the model remains classically disordered at all temperatures. 55

An important feature or the kagome lattice is that the frustrated units (triangles) are arranged to share sites rather than bonds. This precludes the mapping of the kagome ground states to a hardcore dimer model on the dual lattice (known as the diced lattice); this also precludes a mapping to a height model. It is thus an excellent candidate for a disordered quantum magnet. The physics of this model has been discussed in moderate detail in Ref. 16; here, we fill in the missing detail, repeating some material telegraphically for coherence and convenience.

Application of a longitudinal field, $|h|<4 J$, to this magnet leads to the development of a ferromagnetic moment coexisting with a reduced but extensive entropy and critical spin correlations. The result follows in a way formally similar to the $\Gamma=0$ phases in Sect. VII. Each triangle has two up and one down spin, and denoting each down spin by a dimer with endpoints in the centres of the triangles that share it, we obtain a bijective mapping between the ground states and the hardcore dimer covering of the hexagonal lattice; see Fig. 13. This implies, by virtue of the associated height mapping, critical correlations. The entropy per spin, while still nonzero, is reduced to the value of $\mathcal{S}=\mathcal{S}_{\text {tri }} / 3=0.108$; the difference to the triangular lattice arises from the fact that the number of spins per dimer is different on account of the inequivalent mappings used to arrive at the dimer model.

We emphasize that this result is rather unusual. Starting from a disordered magnet, we obtain a critical state with nonzero moment and extensive entropy upon appli- cation of an infinitesimal field; these properties, including the criticality, persist for a finite range of field strengths.

Next, consider applying a small transverse field, $\Gamma \ll$ $|h|$, in addition to the longitudinal one $(0<|h|<4 J)$. The resulting perturbation theory, controlled by the parameter $|\Gamma / h|$, has the following structure. The groundstate condition of the exchange term of the Hamiltonian imposes the restriction of having either one or two down spins ('dimers') per triangle. Starting from a configuration which is also a ground state of the longitudinal field part of the Hamiltonian, we are thus allowed to add dimers violating the hardcore condition, as long as three never meet in one site. To connect different ground states requires relocating three dimers (denoted by crosses in Fig. 13), since the shortest closed loop of bonds of the hexagonal lattice has length six. The lowest-order offdiagonal matrix elements thus arise to $O\left((\Gamma / h)^{6}\right)$, and are precisely those described by the quantum dimer resonance term in Eq. 5.1. This being the unique shortest closed loop, all other terms up to and including sixth order are diagonal. These terms correspond to putting down and then removing up to three dimers. Due to the local structure of the dimer states, the number of such operations, and the concomitant energy denominators, are found to be the same for all ground states. Hence, the diagonal energy shift is uniform and does not generate a lifting of the classical degeneracy.

The ordering pattern is therefore determined by the hexagonal QDM (Eq. 5.1) at $v=0$ and $t \propto \Gamma(\Gamma / h)^{5}$. In dimer language, it is the one depicted in Fig. 7 .

Next, consider the kagome IAFM in a transverse field $\Gamma$ with $h=0$. Following our previously described strategy, we look for a symmetry-breaking pattern within a Landau-Ginzburg-Wilson analysis; However, this meanfield treatment predicts an infinite number of zero modes, corresponding to the simultaneous softening of an entire branch of excitations.57 For Ising spins, high-temperature series expansion studies of the kagome IAFM indicate that thermafluctuations fail to select a wavevector to any order. 58,8

The variational maximally flippable configurations turn out to be the maximally polarised dimer configuration defined above for $h>\Gamma=0$, of which there is an exponentially large number. The resulting hierarchical states are those in which each triangle has a spin pointing up, one pointing down and one pointing along the transverse field. Evidently, these states map onto the three state Potts model on the kagome lattice, which is also known to have a nonzero entropy $\mathcal{S}$.

All these arguments portray the kagome TFIM as a system extremely reluctant to order. We have checked this explicitly by quantum Monte Carlo simulations and found that the TFIM mirrors the classical model, with correlations somewhat enhanced compared to the classical ones, but still rapidly exponentially decaying (see Fig. 3 of Ref. 16). Here, we supplement these data by displaying, in Fig. 14, that the simulated correlation functions have settled down with respect to both quantum 
temperature and discretisation error. Note that in both cases the correlations are extremely small in magnitude below the first few neighbours, in marked contrast to the situation in models known to order; for example, in the triangular IAFM the saturated correlation function remains above 0.5 in these units at the largest distances.

In Fig. 15, we also show that, for a small longitudinal field applied in addition to the transverse one, the rapid decay of the correlation functions remains unaffected, the main effect being the appearance of a net moment visible in the correlations at large distances. The size of this moment is close to linear in $h$, as one expects for a quantum paramagnet. At short distances, we observe the emergence of weak correlations reminiscent of the dimer crystal described above. We therefore conjecture that with further field-tilting results in a continuous quantum phase transition to the ordered dimer phase. We have not been able to confirm this numerically as our simulations fail to equilibrate before the critical value of $h$ is reached.

In Fig. 13 we display the simplest phase diagram for the kagome IAFM in longitudinal and transverse fields consistent with the discussion here, noting that details associated with the tilted-field phase line remains a topic for future study. We close this section by restating its main result, namely the fact that the kagome TFIM is a quantum disordered magnet.

\section{THE SAWTOOTH CHAIN}

An extreme and amusing example of a quantum spin liquid is provided by the sawtooth chain (Fig. 16). It is the ultimate cooperative paramagnet: the locations of the frustrated bonds on each triangle are entirely independent, providing a ground-state entropy $\mathcal{S}=(\ln 3) / 2$ per spin. This is a result of the absence of closed loops of triangles, and the fact that, as in the case of the kagome lattice, they are arranged to share corners.

This chain has been studied in detail by Priour et al. 4 using a high-order series expansion in $J / \Gamma$, which compared favourably with exact diagonalisations. They found no phase transition at any value of $J / \Gamma$, implying that the chain is in a quantum paramagnetic state.

It has been suggested that the ground-state topology plays an important fqle in determining the ordering properties of a magnet.59 It therefore may be of interest to note that this chain has a completely connected groundstate manifold. The proof proceeds by explicit construction of a path from any ground state configuration to a reference ground state configuration, pictured in Fig. 16. First, one picks any down spin on a bottom row (if there are none, any one can be flipped). The remaining two spins of the triangle can be made to agree with the reference state by one of the three operations depicted below the reference configuration; this is then repeated for the neighbouring triangle. In the case of the operation pic- tured on the left, nothing has to be done. A question mark means that the spin can have either orientation and can thus be made to agree with the reference orientation immediately. Once this has been done, the spins enclosed by the ellipse can be oriented appropriately.

\section{THE FULLY FRUSTRATED HEXAGONAL LATTICE}

\section{A. The classical model}

The fully frustrated hexagonal Ising magnet is obtained from the ferromagnetic Ising model on that lattice by changing the sign of one interaction in each hexagon, as depicted in Fig. 17. Since the lattice is bond-sharing, the classical ground states can be again be represented by hardcore dimer coverings of the dual lattice, which is the triangular lattice. Unlike the triangular lattice, the fully frustrated hexagonal lattice does not admit a height model of the types encountered for other lattices we discuss. Although it is possible to give a rule to assign heights to the sites of the hexagonal plaquette, these rules cannot be consistently given for all plaquettes of the full lattice since they involve assigning opposite orientations to neighbouring plaquettes, which is impossible as the dual triangular lattice is not bipartite.

It is found that the classical correlations of the hexagonal FFIM are disordered rather than critical, and the ground-state entropy of the magnet has been evaluated to give $\mathcal{S}=0.21460$

\section{B. Action of the transverse field and flippability analysis}

Let us now consider the dynamics induced by the transverse field. Note that the odd coordination of the lattice precludes any spin from being flippable individually since it cannot have an equal number of frustrated and satisfied bonds. The shortest allowed dimer move consists of moving two occupied dimers and implies flipping a pair of neighbouring spins. Not absolutely all local moves within the ground-state manifold, however, can be generated with these pair spin flips. In fact, one particular ('staggered') configuration exists which does not allow any two spin flips but which instead permits the four dimer move as depicted in Fig. 17.

For infinitesimal $\Gamma$, the ground-state degeneracy is thus lifted to second order in $\Gamma / J$, and the relevant spin flips are the pair flips. To identify the maximally flippable configurations, we note that each dimer can be part of at most two flippable pairs. Since the total number of dimers is fixed, the maximally flippable configurations are those in which each dimer belongs to two pairs.

All maximally flippable configuration can be obtained by carrying out any number of either operation $\mathrm{A}$ or $\mathrm{B}$ 
(right panel of Fig. 17) on a particular, maximally flippable ('columnar') configuration, in addition the symmetry operations of global rotations and global inversion. These operations involve exchanging empty and occupied dimers on an infinite alternating sequence, along a string (A) or a sawtooth (B). They generate, for a system containing $L^{2}$ spins, a number of configurations exponential in $L$ rather than $L^{2}$. The configurations generated by operation $\mathrm{A}$ all incorporate long-range order in one special direction.

\section{Large $\Gamma$ analysis}

Next, we carry out the large $\Gamma$ analysis for this lattice. We first identify the soft modes, then construct the Landau-Ginzburg-Wilson action which we minmise in order to obtain spin configurations and correlations functions.

Since the hexagonal FFIM has four sublattices (labelled as in Fig. 17), the Fourier transform of the interaction matrix is of size $4 \mathrm{x} 4$, and is given by (omitting an overall factor of $J / 2$ :

$$
\left(\begin{array}{cccc}
0 & 1-e^{i q_{x}} & -e^{i q_{y}} & 0 \\
1-e^{-i q_{x}} & 0 & 0 & -e^{-i q_{x}} \\
-e^{-i q_{y}} & 0 & 0 & -1-e^{-i q_{x}} \\
0 & -e^{i q_{x}} & -1-e^{i q_{x}} & 0
\end{array}\right)
$$

The square of the eigenvalues, $\lambda$, are

$$
3 \pm \sqrt{6+2 \cos \left(2 q_{x}\right)-2 \cos \left(q_{x}+q_{y}\right)+2 \cos \left(q_{x}-q_{y}\right)}
$$

The four extremal eigenvalues of interest are $\sqrt{6}$, which occur at wavevectors $\pm(\pi / 6, \pi / 2), \pm(5 \pi / 6, \pi / 2)$. The corresponding eigenvectors occur in complex conjugate pairs:

$$
\begin{aligned}
& \mathbf{v}_{1}=\mathbf{v}_{3}^{*}=\left(\begin{array}{c}
\exp (5 \pi i / 12) / F \\
\exp (-\pi i / 6) / F \\
\exp (-\pi i / 12) \\
1
\end{array}\right) \exp \left(\frac{\pi i}{6} x+\frac{\pi i}{2} y\right) \\
& \mathbf{v}_{2}=\mathbf{v}_{4}^{*}=\left(\begin{array}{c}
\exp (\pi i / 12) \\
\exp (-5 \pi i / 6) \\
\exp (-5 \pi i / 12) / F \\
1 / F
\end{array}\right) \exp \left(\frac{\pi i}{6} x+\frac{\pi i}{2} y\right)
\end{aligned}
$$

where $F=2 \sin (5 \pi / 12)$.

In order to determine the terms in the LGW Hamiltonian, one determines how these modes transform among themselves under the symmetry operations (translations $\left(\mathrm{T}_{\mathrm{x}}, \mathrm{T}_{\mathrm{y}}\right)$, reflection (Ref) and rotation (Rot)) of the underlying lattice. Each of these symmetry operations comes with a gauge transformation since the unit cell of the lattice and the unit cell of the interactions are unequal. These are shown in Fig. 18. The transformation matrices for the amplitudes of these modes under the abovementioned symmetry operations are thus:

$$
\begin{aligned}
& \mathrm{T}_{\mathrm{x}}=\left(\begin{array}{cccc}
e^{i \pi / 6} & 0 & 0 & 0 \\
0 & e^{5 i \pi / 6} & 0 & 0 \\
0 & 0 & e^{-i \pi / 6} & 0 \\
0 & 0 & 0 & e^{-5 i \pi / 6}
\end{array}\right) \\
& \mathrm{T}_{\mathrm{y}}=\left(\begin{array}{cccc}
0 & 0 & 0 & e^{-i \pi / 12} \\
0 & 0 & e^{-5 i \pi / 12} & 0 \\
0 & e^{i \pi / 12} & 0 & 0 \\
e^{5 i \pi / 12} & 0 & 0 & 0
\end{array}\right) \\
& \operatorname{Ref}=\frac{1}{\sqrt{2}}\left(\begin{array}{cccc}
0 & i & e^{i \pi / 4} & 0 \\
-i & 0 & 0 & e^{i \pi / 4} \\
e^{-i \pi / 4} & 0 & 0 & -i \\
0 & e^{-i \pi / 4} & i & 0
\end{array}\right) \\
& \operatorname{Rot}=\frac{1}{\sqrt{2}}\left(\begin{array}{cccc}
0 & e^{i \pi / 12} & e^{-i \pi / 6} & 0 \\
e^{-7 i \pi / 12} & 0 & 0 & e^{i \pi / 6} \\
e^{i \pi / 6} & 0 & 0 & e^{-i \pi / 12} \\
0 & e^{-i \pi / 6} & e^{7 i \pi / 12} & 0
\end{array}\right)
\end{aligned}
$$

To find the eventual action, one has to determine the terms at each order which remain invariant under these transformations. One obtains:

$$
\begin{aligned}
\mathcal{L}= & \left(r+q^{2}\right)\left(\psi_{a}^{2}+\psi_{b}^{2}\right)+u_{4}\left(\psi_{a}^{2}+\psi_{b}^{2}\right)^{2}+ \\
& +u_{6}\left(\psi_{a}^{2}+\psi_{b}^{2}\right)^{3}+ \\
& +v_{6}\left(\psi_{a} \psi_{b}^{5} \cos \left(\theta_{a}-5 \theta_{b}\right)+\psi_{a}^{5} \psi_{b} \cos \left(5 \theta_{a}-\theta_{b}\right)\right)
\end{aligned}
$$

Here we have transformed the four mode amplitudes $\left\{a_{i} \mid i=1 \ldots 4\right\}$ into complex numbers $\psi_{a, b} \exp \left(i \theta_{a, b}\right)$ with $\psi_{a} \exp \left(i \theta_{a}\right)=a_{1}+i a_{3}$ and $\psi_{b} \exp \left(i \theta_{b}\right)=a_{2}+i a_{4}$.

The terms which survive are $O(4)$-symmetric up to sixth order, where in addition a symmetry-breaking term appears. To minimise this action, we use the $O(4)$ symmetric terms to determine $\psi_{a}^{2}+\psi_{b}^{2}$, the radius $R$ of the $O(4)$-sphere, which sets the overall amplitude of the spin pattern. The symmetry-breaking $v_{6}$-term then selects 48 points on this sphere. One finds that $\psi_{a}=\psi_{+}$and $\psi_{a}=\psi_{+} / F ; \theta_{a, b}=0( \pm \pi / 6)$ for $v_{6}<(>) 0$. The other 47 solutions are generated by the operations $\psi_{a} \leftrightarrow \psi_{b}$ and $\left\{\theta_{a} \rightarrow \theta_{a}+\pi / 12, \theta_{b} \rightarrow \theta_{b}+5 \pi / 12\right\}$. The 48 solutions for a given sign of $v_{6}$ are related by simple symmetry operations. Note the large unit cell of the ordering pattern, which contains 48 spins. This can in part be attributed to the non-uniform pattern of bonds. In the gauge-invariant dimer language, the unit cell could be smaller; an example of this is given further down.

The correlation functions obtained from the above expressions are for soft spins so that they will certainly not be quantitatively found in the real system at low temperatures. However, their qualitative features, if the ordering pattern is correctly predicted, should survive. These are peaks at the appropriate wavevectors in Fourier space; due to the factors of $1 / F$ for the amplitudes $\psi_{a, b}$, these peaks are not expected to have equal heights for correlation functions for spins on the same sublattice. It turns out that the calculated correlation functions when, averaged over all 48 minima, are the same as those averaged over the entire $O(4)$ sphere.

We have looked for this ordering pattern by quantum Monte Carlo. There are several features complicat- 
ing this search. One arises from the quantum dynamics which takes the form of double spin flips. Luckily, one is saved here by the fact that the cluster algorithm can be generalised to this case. The most simple-minded extension to double spin flips would have been to generate two neighbouring clusters (rods in the imaginary time direction) separately - by design, the cluster algorithm would cancel the problematic Boltzmann factor $\exp \left(-K^{\tau} / 2\right)$ in each of the two clusters separately. However, clusters of unequal height, which become overwhelmingly probably for large $K^{\tau}$, cannot be flipped because permissible moves must be double spin flips in any and all planes. The way around this is to construct a cluster as a pair of rods. This works because the cluster can either terminate when encountering zero, one or two domain walls in the time direction. The case of zero and two can be taken care of by working with an effectively doubled coupling in the time direction; the case of one domain wall takes care of itself as there is no Boltzmann factor to be canceled in the first place. This prescription can therefore not be generalised to other multiple-spin flips in a straightforward manner.

Another problem is the very large unit cell of the ordering pattern, which is 48 spins but only fits into periodic boundary conditions for system sizes multiples of 192 spins. We have thus not attempted to do a complete finite-size scaling study and have contented ourselves with displaying the presence of peaks at the wavevectors predicted above.

In Fig. 19 we have displayed the correlation function for spins on sublattice 4 along the $x$ and $y$ directions (also see Fig. 17). A peak at the expected location is clearly visible. This suggests that there is at least significant short-range order of the predicted nature present in this regime. We caution, however, that we have not been able to do a complete numerical analysis of this problem that would unambiguously establish this ordering pattern.

To rationalise the above results, we finally present a dimer pattern which correspouds to a spin pattern of the correct size unit cell (Fig. 20). 34 We have not found a way of relating the spin-spin correlations precisely to those of the solutions of the LGW theory found above, but one can see rather nicely how the periodicity of twelve and four in the $x$ and $y$ directions comes about from a smaller size unit cell in the gauge invariant description. The state pictured is favourable because each spin fluctuates in a pair gaining energy from the transverse field, and because there are many equivalent such configurations, as explained in the caption.

\section{SUMMARY}

In this paper we have described our analysis of a number of frustrated Ising systems with a quantum dynamics introduced by a magnetic field applied transverse to the Ising axis. We have argued that these models are of theoretical interest as the simplest settings in which quantum dynamics interacts with classical frustration. They can represent effective theories of systems, such as short ranged RVB magnets, where the low energy dynamics contains a frustrated Ising degree of freedom and are likely of experimental interest when suitable materials are probed by the application of a transverse magnetic field.

We have been able to make considerable progress in elucidating the phase structure of these systems which sheds much light on the interplay between the structure of the macroscopic degeneracy and the quantum dynamics. Our results include instances of "order by disorder" and of "disorder by disorder" (i.e. Ising spin liquids). In arriving at these we have used two systematic approaches: a variational approach that builds on the local entropy of different classical configurations that is the Ising analog of semiclassical analyses of magnets with continuous symmetry, and an LGW analysis that attempts to guess at the large order structure of an expansion about the large transverse field paramagnet. We have also made use of quantum Monte Carlo simulations as well as of mappings to height and dimer models with the latter allowing a connection to RVB physics.

There appear to be several directions that can be pursued further. Along the lines of the questions addressed in this paper, the analysis of the kagome system in tilted fields and a definitive analysis of the hexagonal lattice problem are needed. Further, he structure and energetics of the low energy excitations needs to be worked out to have an understanding of the dynamics at low temperatures. Beyond this it would be interesting to include the dynamics of the transverse exchange and see what happens both in the approach to the Heisenberg limit and when a transverse field is also present. We expect such work to be both fruitful and instructive.

\section{ACKNOWLEDGEMENTS}

We are very grateful to $\mathrm{P}$. Chandra for collaboration on many aspects of this work. We would also like to thank M. Aizenman, J. Chalker, C. Henley, D. Huse, S. Kivelson, E. Lieb and S. Sachdev for useful discussions. This work was supported in part by grants from the Deutsche Forschungsgemeinschaft, the NSF (grant No. DMR-9978074), the A. P. Sloan Foundation and the David and Lucille Packard Foundation. 
${ }^{1}$ G. H. Wannier, Phys. Rev. 79, 357 (1950).

${ }^{2}$ R. M. F. Houtappel, Physica 16, 425 (1950).

${ }^{3}$ In truth, the issue is somewhat delicate in this particular case, with the zergtemperature limit of the spin correlations being critical 1 A better example is the kagome lattice Ising antiferromagnet (Fig. 2); we will return to these subtleties below.

${ }^{4}$ J. Stephenson, J. Math. Phys. 11, 413 (1970); J. Math. Phys. 11, 420 (1970).

${ }^{5}$ J. Villain, Z. Phys. B 33, 31 (1979).

${ }^{6}$ For a review, see: R. Moessner, cond-mat/0010301.

${ }^{7}$ We should note that there is considerable laxity in the literature regarding the meaning of the term "frustrated magnet" even for classical systems. It would be best to restrict it to systems that have an extensive entropy or ground state dimensionality at $T=0$ but it is not unusual to use it to describe all systems with bond interactions that are not simultaneously satisfiable. An instance of the latter, but not of the former is the triangular lattice Heisenberg model. We should also note that even with the more restricted usage it is not synonymous with absence of ordering - a constrained four states Potts model on the square lattice (J. K. Burton and C. L. Henley, cond-mat/9708171) appears to be an example of an ordered system with extensive entropy at $T=0$, as is a three-leg ladder that we discuss below.

${ }^{8}$ D.A. Huse and A.D. Rutenberg, Phys. Rev. B 45, 7536 (1992).

9 J. Villain, R. Bidaux, J. P. Carton and R. J. Conte, J. Phys. - Paris 41, 1263 (1980).

${ }^{10}$ E. F. Shender, Sov. Phys. JETP 56, 178 (1982).

${ }^{11}$ For a review, see: M. F. Collins and O. A. Petrenko, Can. J. Phys. 75, 605 (1997).

12 see, e.g., J. T. Chalker and J. F. G. Eastmond, Phys. Rev. B 46, 14201 (1992); C. Zeng and V. Elser, Phys. Rev. B 51, 8318 (1995); P. Lecheminant, B. Bernu, C. Lhuillier, L. Pierre and P. Sindzingre, Phys. Rev. B 56, 2521 (1997); F. Mila, Phys. Rev. Lett. 81, 2356 (1998) and references therein.

${ }^{13}$ P. W. Anderson, Mat. Res. Bull. 8, 153 (1973); P. Fazekas and P. W. Anderson, Phil. Mag. 30, 23 (1974).

${ }^{14}$ In the future we hope to explore other instances of single spin quantum dynamics involving either larger values of spin or the extension of classical continuous spin models to quantum rotor models. The quantum dynamics of mobile holes in a frustrated Ising background is discussed in Ref. 15.

${ }^{15}$ R. Moessner and S. L. Sondhi, to appear in Phys. Rev. B.

${ }^{16}$ R. Moessner, S. L. Sondhi and P. Chandra, Phys. Rev. Lett. 84, 4457 (2000).

${ }^{17}$ For some work on one-dimensional models, see B.K. Chakrabati, A. Dutta and P. Sen, Quantum Ising Phases and Transitions in Tranverse Ising Models, (SpringerVerlag, Berlin, 1996).

18 S. Sachdev, Quantum Phase Transitions (Cambridge University, Cambridge, 2000).

19 D. S. Fisher, Phys. Rev. Lett. 69, 534 (1992).

${ }^{20}$ G. Aeppli and T.F. Rosenbaum in Dynamical Properties of Unconventional Magnetic Systems, A.R. Skjeltorp and D. Sherrington eds., (Kluwer Academic Publishers, Amsterdam, 1998).
${ }^{21}$ P. W. Anderson, Science 235, 1196 (1987).

${ }^{22}$ D. S. Rokhsar and S. A. Kivelson, Phys. Rev. Lett. 61, 2376 (1998).

${ }^{23}$ N. Read and S. Sachdev, Nucl. Phys. B 316, 609 (1989); Phys. Rev. Lett. 66, 1773 (1991).

24 X.G. Wen, Phys. Rev. B 44, 2664 (1991).

${ }^{25}$ C. Mudry and E. Fradkin, Phys. Rev. B 49, 5200 (1994); Phys. Rev. B 50, 11409 (1994).

${ }^{26}$ S. Sachdev and M. Vojta, J. Phys. Soc. Jpn. 69, 1 (2000)

27 T. Senthil and M. P. A. Fisher, Phys. Rev. B 62, 7850 (2000).

${ }^{28}$ R. Moessner and S. L. Sondhi, cond-mat/0007378.

${ }^{29}$ M. Suzuki, Prog. Theor. Phys. 46, 1337, (1971); ibid 56, 2454 (1976).

${ }^{30}$ H.F. Trotter, Proc. Am. Math. Soc. 10, 545 (1959).

${ }^{31}$ R. H. Swendsen, J. S. Wang and A. M. Ferrenberg, Top. Appl. Phys. 71, 75 (1992)

${ }^{32}$ H. Rieger and N. Kawashima, Eur. Phys. J. B 9, 233 (1999)

${ }^{33}$ We note that the existence of a dimer mapping has interesting topological consequences, 22 which however will not be discussed in this work.

${ }^{34}$ We thank David Huse for a useful discussion of this point.

35 This is related to the fact that in a layered, ferromagnetically stacked frustrated magnets, fluctuations will determine which state is selected as ground state in the limit $T \rightarrow 0$. The importance of fluctuations for such stacked magnets was pointed out by S. N. Coppersmith, Phys. Rev. B 32, 1584 (1985). In our formulation, fluctuations are naturally included, and the mapping to the stacked magnets provides a formal connection between these results.

${ }^{36}$ More precisely, the graph on which hopping is occurs can be defined as a high dimensional but finite lattice. For a system of $N$ spins, it is a subgraph of an $N$ dimensional hypercube.

${ }^{37}$ R. Moessner and J. T. Chalker, Phys. Rev. Lett. 80, 2929 (1998); Phys. Rev. B 58, 12049 (1998).

38 D. Blankschtein, M. Ma, A.N. Berker, G.S. Grest and C.M. Soukoulis, Phys. Rev. B 29, 5250 (1984).

${ }^{39}$ D. Blankschtein, M. Ma and A.N. Berker, Phys. Rev. B 30, 1362 (1984).

${ }^{40}$ D.J. Priour Jr, M. P. Gelfand and S. L. Sondhi, condmat/0005185.

${ }^{41}$ Note that the columnar dimer state is disordered in dimer language as it breaks no symmetries of the lattice. Had the hierarchical state been selected, there would have been breaking of translational symmetry.

${ }^{42}$ M. H. Waldor, W. F. Wolff and J. Zittartz, Phys. Lett. A 106, 261 (1984); Z. Phys. B 59, 43 (1985).

${ }^{43}$ H.W.J. Blöte and H.J. Hilhorst, J. Phys. A15, L631 (1982); B. Nienhuis, H.J. Hilhorst and H.W. Blöte, ibid 17, 3559 (1984).

${ }^{44}$ We note that this specific problem has been treated by Orland (P. Orland, Phys. Rev. B 47, 11280 (1993)), using a string representation for the associated dimer configurations. Unfortunately there are inconsistencies associated with Orland's string decomposition, and furthermore his chosen boundary conditions on the hexagonal lattice do not accomodate the uniform state. There is also a problem of overcounting associated with the antisymmetrization procedure used, so the status of his results of a gapless spec- 
trum and no fluctuation-selection is unclear. (C. L. Henley, S. Sachdev, N. Read, private communications).

45 This mapping was independently noted by C.L. Henley in A. Gervois, M. Gingold and D. Iagolnitzer, eds. Book of Abstracts of STATPHYS 20 (IUPAP Commission on Statistical Physics, Paris, 1998).

${ }^{46}$ J. V. Jose, L. P. Kadanoff, S. Kirkpatrick and D. R. Nelson, Phys. Rev. B 16, 1217 (1977).

${ }^{47}$ M. E. Fisher, Phys. Rev. 124, 1664 (1961).

${ }^{48}$ R. A. Jalabert and S. Sachdev, Phys. Rev. B 44, 686 (1991).

49 P. Orland, Phys. Rev. B 49, 3423 (1994)

${ }^{50}$ P. W. Leung, K. C. Chiu and K. J. Runge, Phys. Rev. B 54, 12938 (1996).

${ }^{51}$ P. W. Anderson, Phys. Rev. 102, 1008 (1956)

${ }^{52}$ E. H. Lieb, Phys. Rev. 162, 162 (1967).

${ }^{53}$ For a review, see: R. Liebmann, Statistical Mechanics of Periodic Frustrated Ising Systems, (Springer, Berlin, 1986).

${ }^{54}$ Different representations of the ice model are explained in G. T. Barkema and M. E. J. Newman, Phys. Rev. E 57, 1155 (1998)

${ }^{55}$ K. Kano and S. Naya, Prog. Theor. Phys. Japan 10, 158 (1953).

${ }^{56}$ L. Pauling, The Nature of the Chemical Bond, (Cornell University Press, Ithaca, 1938).

57 J. N. Reimers, A. J. Berlinsky and A.-C. Shi, Phys. Rev. B 43, 865 (1991)

58 A.B. Harris, C. Kallin and A.J. Berlinsky, Phys. Rev. B 45, 2899 (1992).

59 A. Suto, Z. Phys. B 44, 121 (1981); W. F. Wolff, P. Hoever and J. Zittartz, Z. Phys. B 42, 259 (1981).

${ }^{60}$ W. F. Wolff and J. Zittartz, Z. Phys. B 44, 139 (1982). 


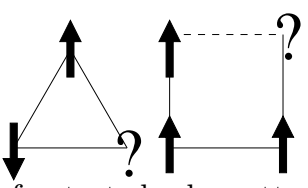

FIG. 1. Two frustrated plaquettes with Ising spins. In the canonical example of the antiferromagnetic triangle, anti-aligning two spins leaves the direction of the third undetermined. Similarly, a square can be frustrated by choosing an odd number of bonds to be antiferromagnetic. For such mixed-bond models, we represent (anti)ferromagnetic bonds by (dashed) solid lines.
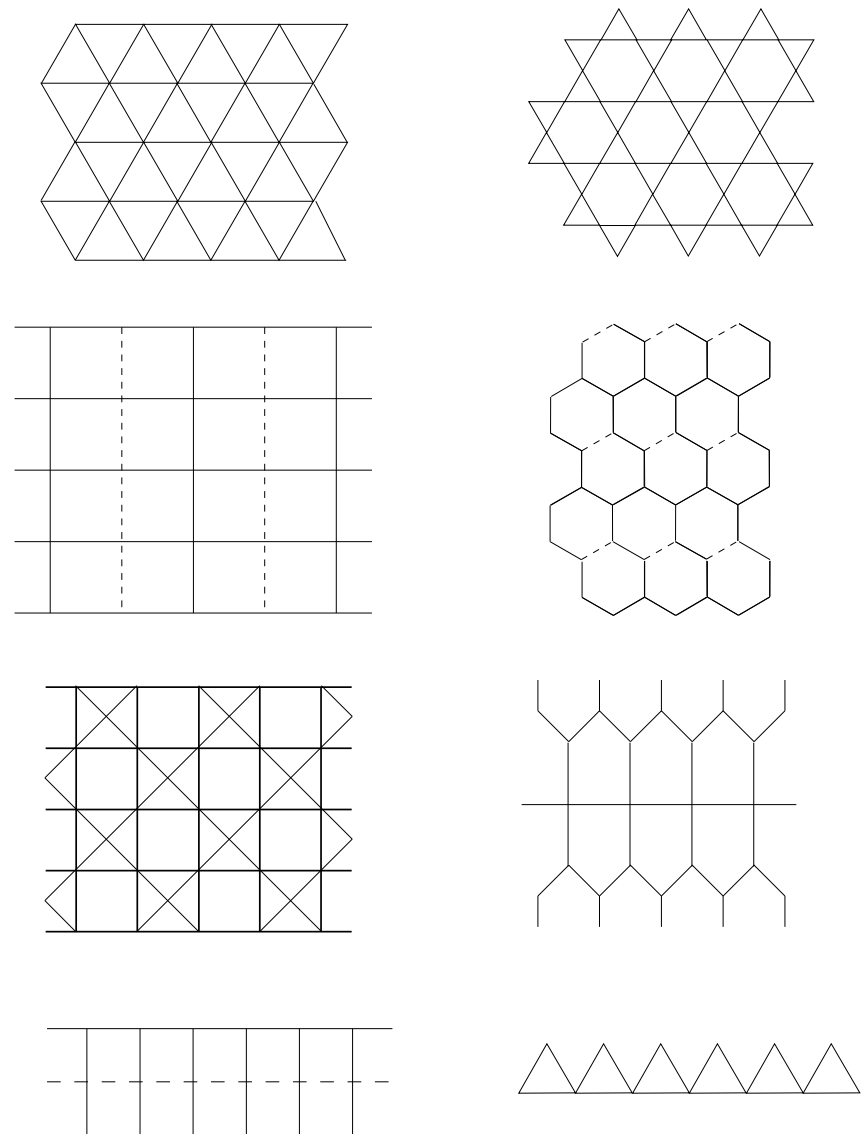

FIG. 2. The lattices on which fully frustrated transverse field Ising models are discussed in this paper. Clockwise from noon: kagome, hexagonal, pentagonal, sawtooth chain, three-leg ladder, square lattice with crossings ("two-dimensional pyrochlore"), square and triangular. For normally unfrustrated lattices, frustration is introduced by choosing an odd number of bonds in each plaquette to be antiferromagnetic (see Fig. 1).
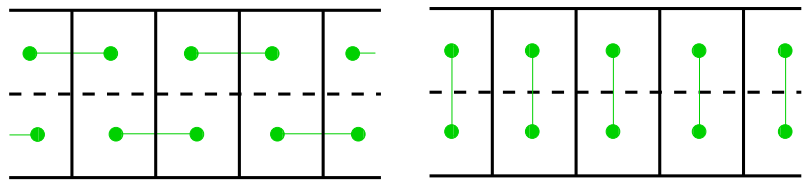

FIG. 3. The fully frustrated three-leg ladder. Solid lines correspond to ferromagnetic bonds, dashed lines to antiferromagnetic ones. Dimer representations of the columnar (right) and one of the two staggered (left) configurations.

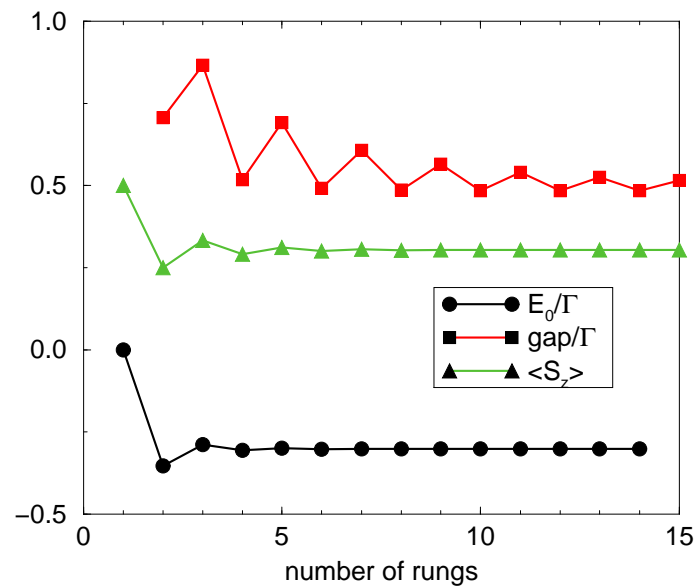

FIG. 4. Ground-state energy (proportional to $\left\langle S_{x}\right\rangle$ ), energy gap and $\left\langle S_{z}\right\rangle$ for the three-leg lader in the limit $\Gamma \rightarrow 0^{+}$ from exact diagonalisation.

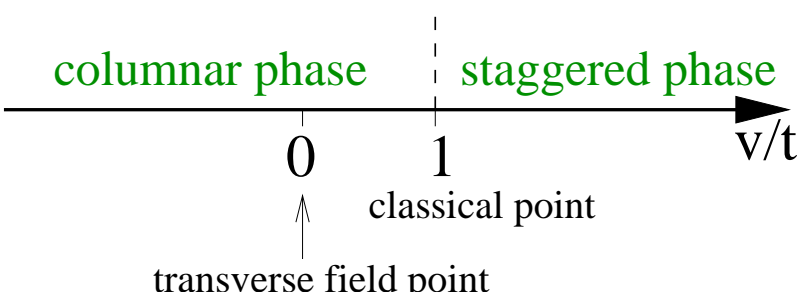

FIG. 5. The phase diagram of the Rokhsar-Kivelson quantum dimer model for the fully frustrated Ising magnet on the three-leg ladder. The diagonal correlations at $v=t$ are those of the classical dimer model. The infinitesimal transverse field problem, $\Gamma=0^{+}$, maps to the point $v=0$ and thus corresponds to a finite jump of $v$.

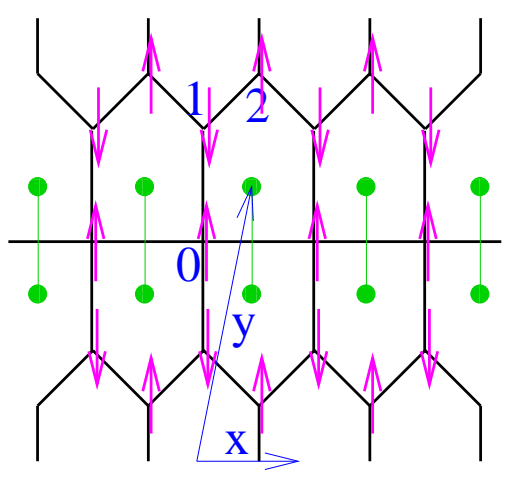


FIG. 6. The pentagonal lattice with its maximally flippable configuration. The three spins in the unit cell are labelled by $(0,1,2)$, and the $x$ and $y$-lattice translation vectors are given by the arrows. The leading-order perturbation theory reduces to studying the central horizontal ladder, which is equivalent to the three-leg ladder of Fig. 3

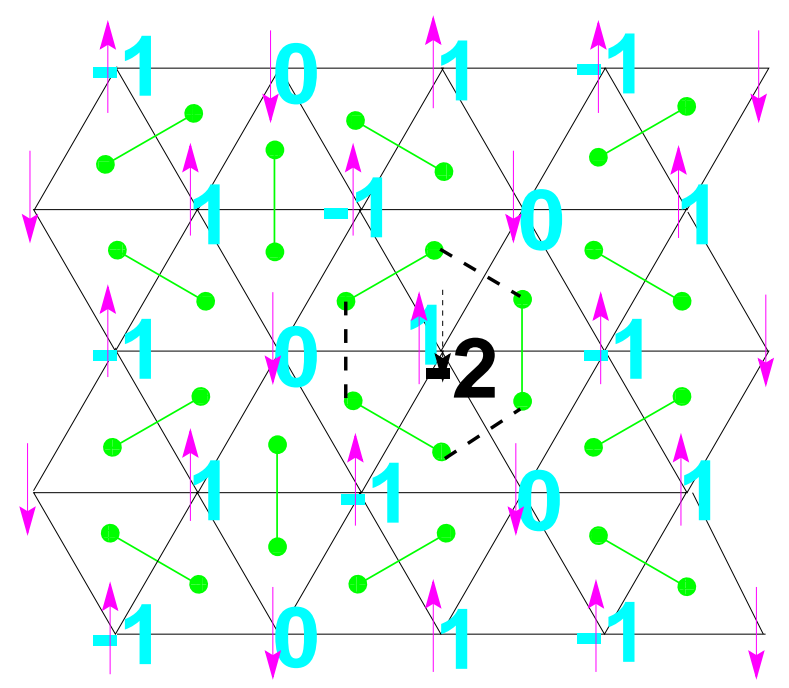

FIG. 7. The maximally flippable spin state (arrows) and its corresponding height and dimer configurations on the triangular lattice. The result of a single spin flip for the dimer and height configurations is also shown. See the text for details.

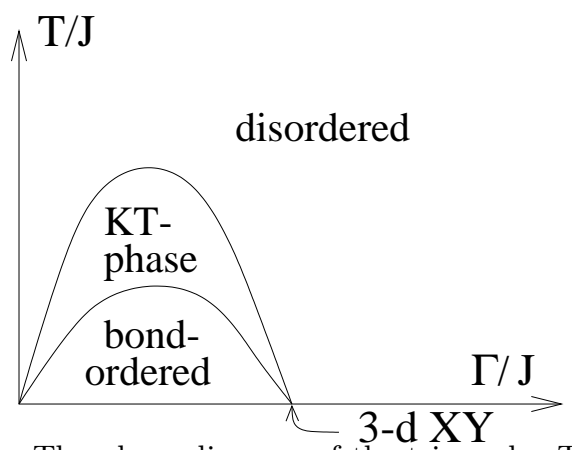

FIG. 8. The phase diagram of the triangular TFIM. The pattern of the ordered phase is depicted in Fig. 目

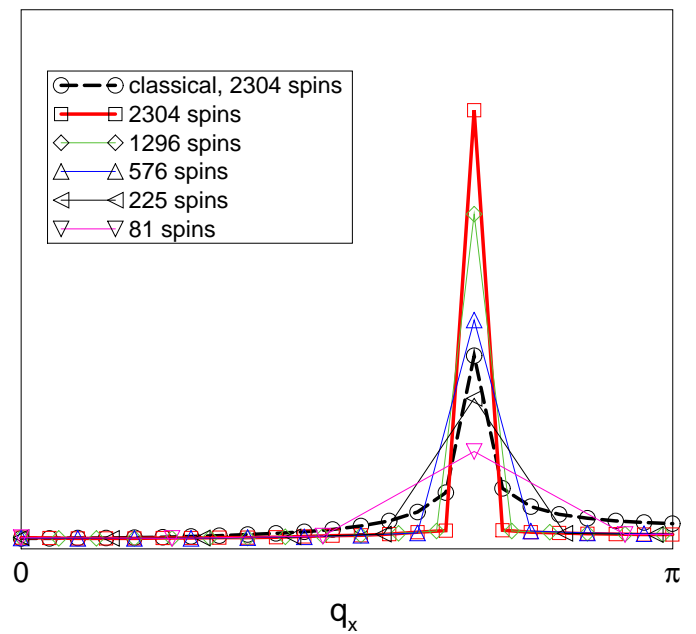

FIG. 9. The absolute value of the Fourier transform of the spin-spin correlation function along the $x$-direction, $\langle S(x) S(0)\rangle$, (arbitrary units, at low temperature), as a function of $q_{x}$ ranging from 0 to $\pi$. Note the growing and narrowing Bragg peak.

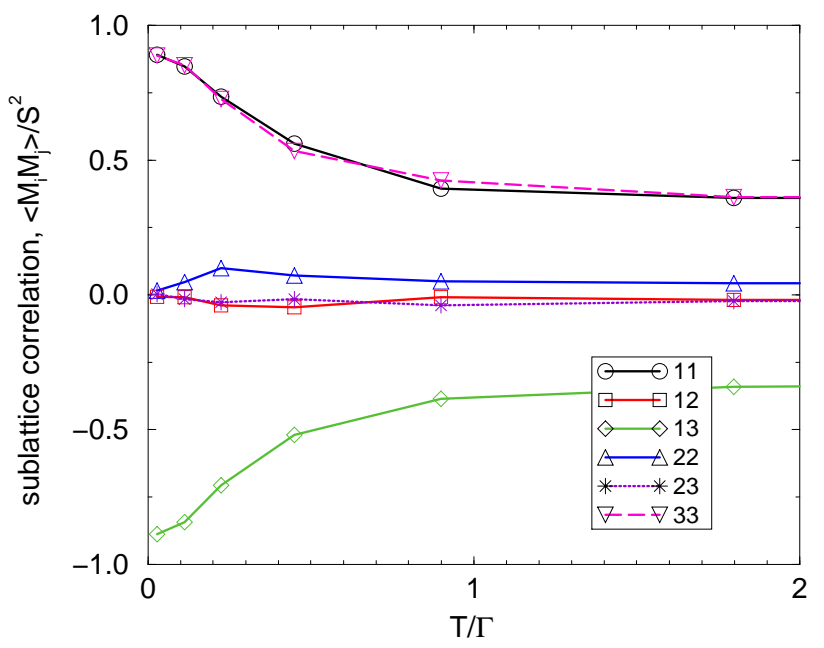

FIG. 10. The correlation matrix, $\left\langle M_{i} M_{j}\right\rangle=\left\langle M_{j} M_{i}\right\rangle$ of sublattice magnetisations, $M_{i}$, for a three sublattice state, as a function of the quantum temperature for a system with 729 spins. The sublattices are labelled by the size of their magnetisation so that $M_{1}>M_{2}>M_{3} .\left\langle M_{2}^{2}\right\rangle \simeq 0$ implies that one sublattice has zero root-mean square magnetisation. $\left\langle M_{1}^{2}\right\rangle \simeq\left\langle M_{3}^{2}\right\rangle \simeq-\left\langle M_{1} M_{3}\right\rangle$ implies the other two sublattices have equal and opposite magnetisation. 


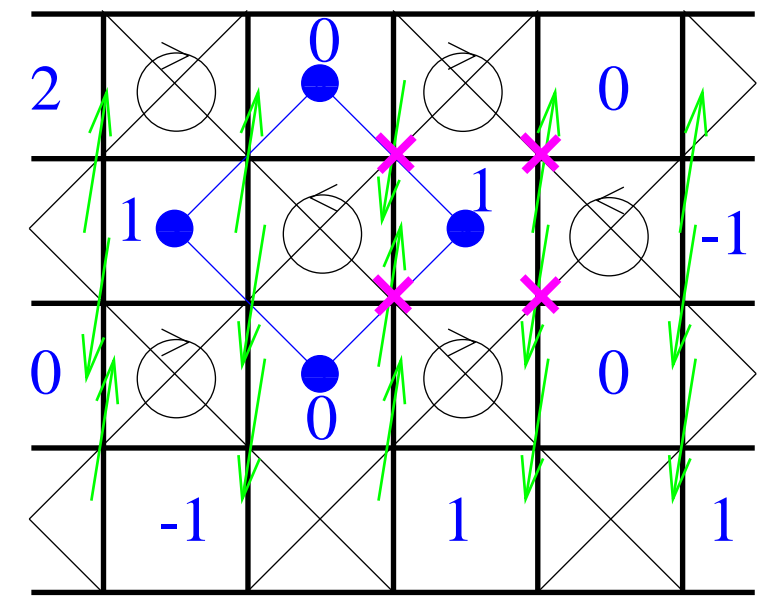

FIG. 11. The square lattice with crossings, or two-dimensional pyrochlore. A spin and the resulting height configuration are shown. Other features are explained in the text.

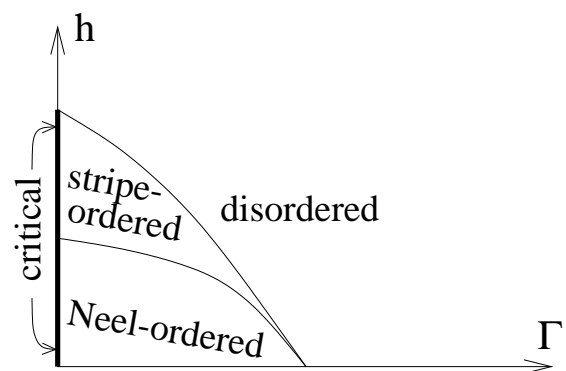

FIG. 12. The phase diagram in the $h-\Gamma$ plane for the square lattice with crossings. The precise extent of either phase away from $\Gamma / h \ll 1$ is unknown.

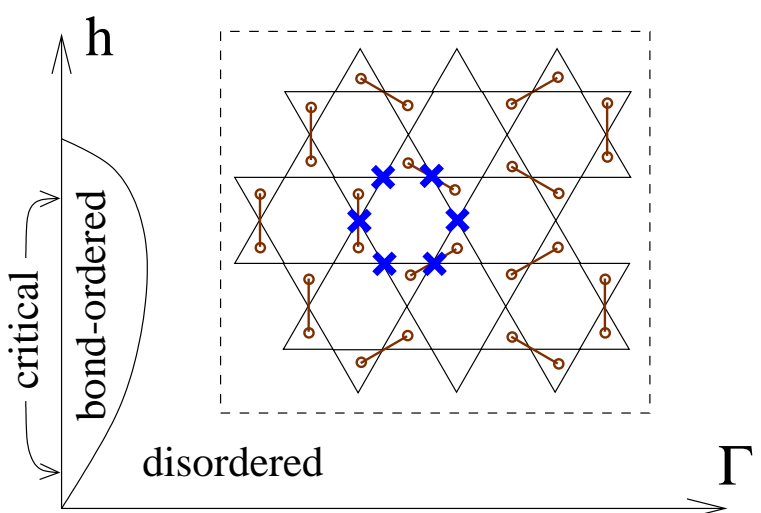

FIG. 13. Phase diagram for the kagome Ising antiferromagnet in a field. Inset: Mapping of the kagome IAFM in a longitudinal field onto the hexagonal lattice dimer model. The down spins are marked by dimers. The non-trivial move to sixth order in perturbation theory corresponds to flipping all the spins marked by crosses.

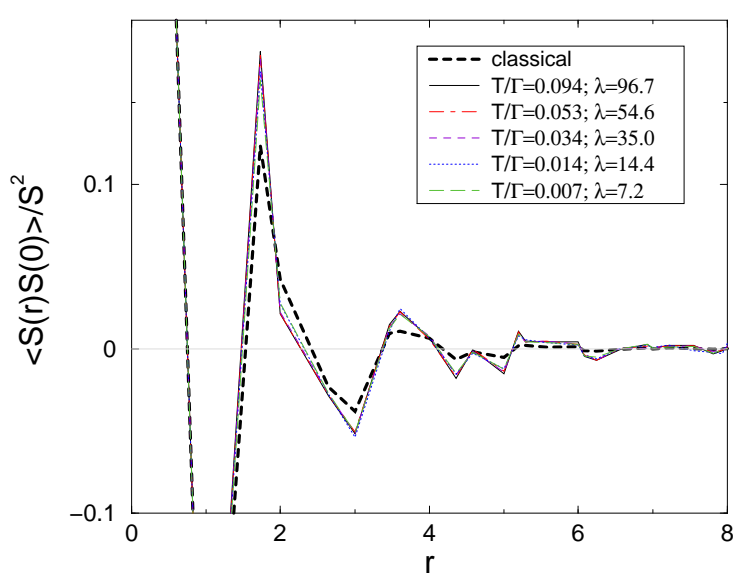

FIG. 14. The angularly averaged spin-spin correlation function for different quantum temperatures and discretisations, $\lambda=\exp \left(K^{\tau} / 2\right)$. Note the rapid decay and the enlarged scale. The quantum curves lie almost on top of one another.

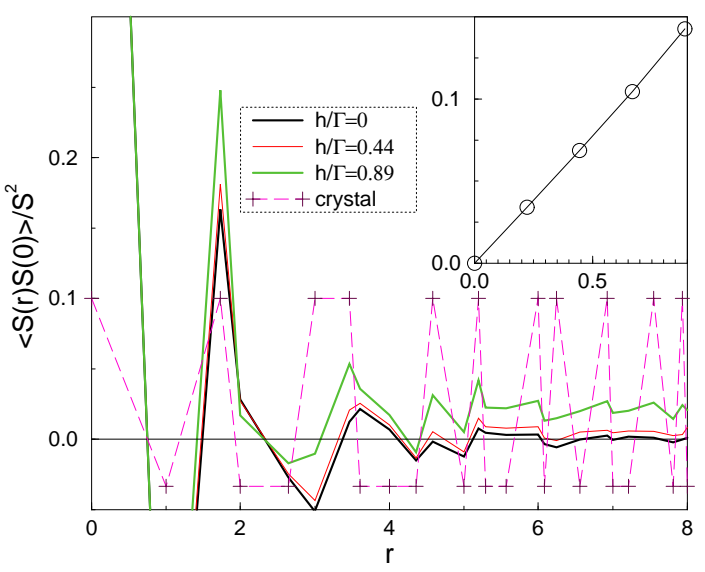

FIG. 15. The angularly averaged spin-spin correlation function for the kagome TFIM in a longitudinal field. The correlations corresponding the dimer crystal (Fig. 13), scaled down by a factor of 10 , are also shown. Note that the high-field curve starts moving towards the crystal correlations at small distances. Inset: The magnetisation per spin (ordinate) is linear in field $(h / \Gamma$, abscissa).

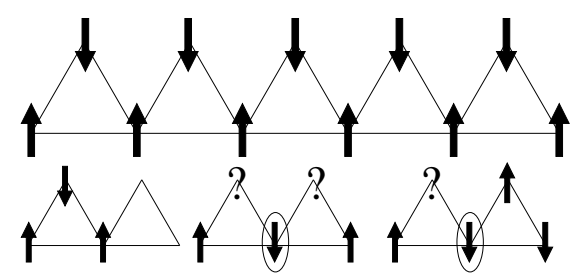

FIG. 16. The sawtooth chain with the reference ground state and the three operations needed for proving ground-state connectedness (see text). 

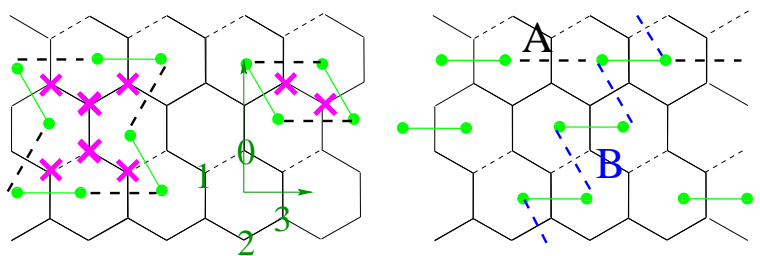

FIG. 17. The fully frustrated hexagonal lattice. The basis spins of the lattice are numbered $0-3$ and the rectangular lattice translation vector in the $x(y)$ direction is given by the horizontal (vertical) arrow. The pair spin flip and a non-trivial multiple spin flip are indicated with their corresponding dimer moves. Maximally flippable states are generated from the columnar seed state by interchanging occupied and empty dimers along horizontal lines (A) or in a sawtooth pattern (B).
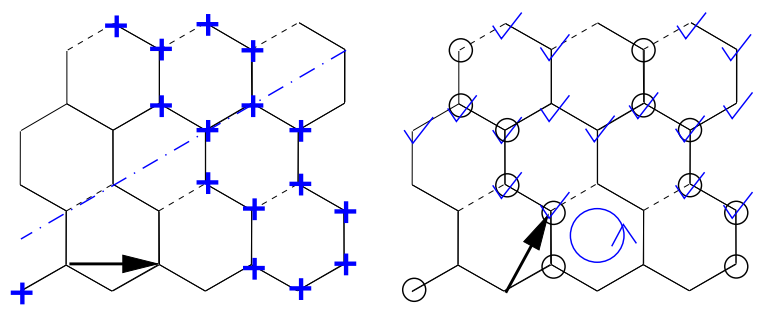

FIG. 18. The symmetry transformations and concomitant gauge transformations for the Landau-Ginzburg-Wilson action. Translation in the $x$-direction (arrow in left panel) require no gauge tranformation. Reflections about the dot-dashed line go along with inversion of the spins marked by crosses; translations by the other hexagonal lattice vector (arrow in the right panel) require flipping the circled spins, and rotations by $\pi / 3$ (circle with arrow) involve flipping the spins denoted by ticks.

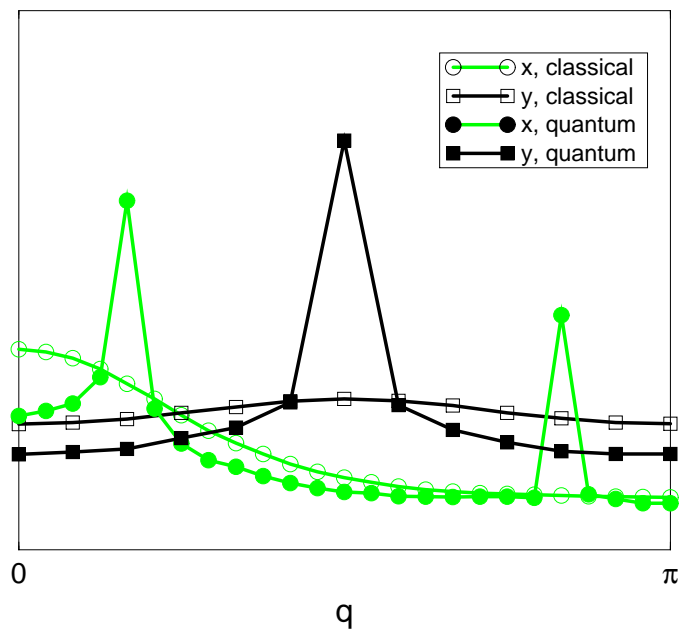

FIG. 19. The Fourier transform of the absolute value of the autocorrelation function of a spin on sublattice 4 in both $x$ and $y$ directions for a system of 4608 spins. Results for the classical and the quantum $\left(\Gamma=0^{+}\right)$cases are shown, with a quantum temperature $T_{Q} / \Gamma=0.028$ nominally, but the discretisation error is rather large: $\lambda=4.5$ only.

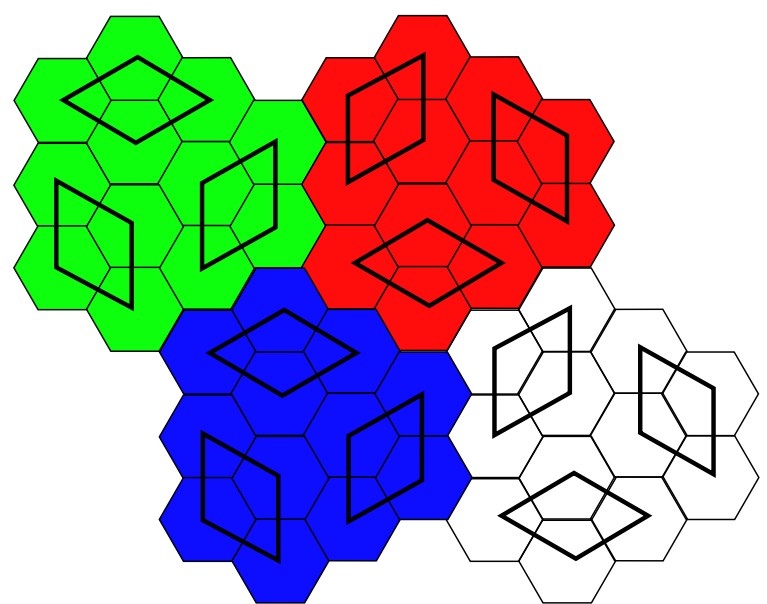

FIG. 20. Cartoon of a dimer pattern with a maximal number of independently flippable pairs of spins. To obtain the corresponding spin pattern, one has to choose a gauge, i.e. one has to make a choice of which bonds are antiferromagnetic. Fig. 17 shows one such choice. For this choice, the spin pattern has a 48 site unit cell (two plaquettes on the left), whereas the gauge invariant dimer description only has 24 sites in a unit cell (any single plaquette). A rhombus stands for a pair of dimers (frustrated bonds) resonating between two configurations, so that the two spins enclosed by it can be thought of as flipping together to take advantage of the transverse field. This pattern cannot be derived from a uniform maximally flippable state but has extensive 'configurational' entropy, as there are two distinct ways (shown in the left and right pairs of plaquettes, respectively) of pairing up the spins in each plaquette. 